\title{
Brain-Wide Mapping of Water Flow Perception in Zebrafish
}

\author{
${ }^{\circledR}$ Gilles Vanwalleghem, ${ }^{1}$ Kevin Schuster, ${ }^{2}$ Michael A. Taylor, ${ }^{3}$ Itia A. Favre-Bulle, ${ }^{1,4}$ and ${ }^{\circledR}$ Ethan K. Scott ${ }^{1}$ \\ ${ }^{1}$ The Queensland Brain Institute, The University of Queensland, St. Lucia, Queensland 4072, Australia, ${ }^{2}$ School of Biomedical Sciences, The \\ University of Queensland, St. Lucia, Queensland 4072, Australia, ${ }^{3}$ Australian Institute of Bioengineering and Nanotechnology, The University of \\ Queensland, St. Lucia, Queensland 4072, Australia, and ${ }^{4}$ School of Mathematics and Physics, The University of Queensland, St. Lucia, Queensland \\ 4072, Australia
}

Information about water flow, detected by lateral line organs, is critical to the behavior and survival of fish and amphibians. While certain aspects of water flow processing have been revealed through electrophysiology, we lack a comprehensive description of the neurons that respond to water flow and the network that they form. Here, we use brain-wide calcium imaging in combination with microfluidic stimulation to map out, at cellular resolution, neuronal responses involved in perceiving and processing water flow information in larval zebrafish. We find a diverse array of neurons responding to head-totail (h-t) flow, tail-to-head (t-h) flow, or both. Early in this pathway, in the lateral line ganglia, neurons respond almost exclusively to the simple presence of $h-t$ or $t-h$ flow, but later processing includes neurons responding specifically to flow onset, representing the accumulated displacement of flow during a stimulus, or encoding the speed of the flow. The neurons reporting on these more nuanced details are located across numerous brain regions, including some not previously implicated in water flow processing. A graph theory-based analysis of the brain-wide water flow network shows that a majority of this processing is dedicated to h-t flow detection, and this is reinforced by our finding that details like flow velocity and the total accumulated flow are only encoded for the h-t direction. The results represent the first brain-wide description of processing for this important modality, and provide a departure point for more detailed studies of the flow of information through this network.

Key words: calcium imaging; lateral line; light sheet microscopy; microfluidic; sensory; zebrafish

Significance Statement

In aquatic animals, the lateral line is important for detecting water flow stimuli, but the brain networks that interpret this information remain mysterious. Here, we have imaged the activity of individual neurons across the entire brains of larval zebrafish, revealing all response types and their brain locations as water flow processing occurs. We find neurons that respond to the simple presence of water flow, and others attuned to the direction, speed, and duration of flow, or the accumulated displacement of water that has passed during the stimulus. With this information, we modeled the underlying network, describing a system that is nuanced in its processing of water flow simulating head-to-tail motion but rudimentary in processing flow in the tail-to-head direction.

\section{Introduction}

The lateral line system provides water flow sensation in fish and amphibians, informing behaviors as varied as noncontact

Received Jan. 5, 2020; revised Mar. 18, 2020; accepted Mar. 20, 2020.

Author contributions: G.V., K.S., M.A.T., I.A.F.-B., and E.K.S. designed research; G.V., K.S., and M.A.T. performed research; G.V. analyzed data; G.V. and E.K.S. wrote the paper.

The authors declare no competing financial interests.

Support for this research was provided by an National Health and Medical Research Council Project Grant (APP1066887) and three Australia Research Council Discovery Project Grants (DP140102036, DP110103612, and DP190103430) to E.K.S., and an EMBO Long-Term Fellowship to G.V.; and a Fellowship from the Human Frontier Science Program to M.A.T. Support was also provided by the Australian National Fabrication Facility, QLD node. We thank the Biological Resources Aquatics Team at the University of Queensland for animal care.

Correspondence should be addressed to Gilles Vanwalleghem at g.vanwalleghem@uq.edu.au or Ethan K. Scott at ethan.scott@uq.edu.au.

https://doi.org/10.1523/JNEUROSCI.0049-20.2020

Copyright $(2020$ the authors assessment, predator evasion, hunting, and rheotaxis (Partridge and Pitcher, 1980; Montgomery et al., 1997; McHenry et al., 2009; Olszewski et al., 2012; Suli et al., 2012; Butler and Maruska, 2015). Its sensory neuromasts are spread across the body of the animal and are composed of bundles of hair cells whose polarized organization makes them direction selective either in the anterior-posterior or dorsal-ventral axis (Dijkgraaf, 1963; Ghysen and Dambly-Chaudière, 2004). Signals from neuromasts are carried to the lateral line ganglia (LLGs), and from there into the neuropil of the medial octavolateral nucleus (MON).

Each afferent neuron in the LLGs selectively innervates hair cells of a given polarity (Nagiel et al., 2008; Faucherre et al., 2009; Ji et al., 2018). As a result, although they may receive input from hair cells spanning multiple neuromasts, afferent neurons are direction selective (Nagiel et al., 2008; Faucherre et al., 2009; Ji et al., 2018). The lateral line system can also be topographically 
divided into the following two regions: the anterior lateral line, comprising cranial neuromasts whose afferent neurons are found in the anterior LLG (Raible and Kruse, 2000), and the posterior lateral line, comprising trunk and tail neuromasts innervated by the posterior lateral line ganglion (pLLG) afferent neurons (Metcalfe et al., 1985; Raible and Kruse, 2000; Schuster et al., 2010).

Tract-tracing studies and single-cell labeling have shown that, in addition to the MON and Mauthner neurons, the afferent neurons from the lateral line ganglia project to the eminentia granularis (McCormick, 1989; Alexandre and Ghysen, 1999; Bleckmann, 2008; Liao and Haehnel, 2012), which further projects to the torus semicircularis (TS), homologous to the inferior colliculus, and the deep layers of the optic tectum (OT), homologous to the superior colliculus (McCormick and Hernandez, 1996; Bleckmann, 2008). Lateral line information is finally conveyed to the telencephalon from the TS via the lateral preglomerular nucleus (Wullimann, 1997; Bleckmann, 2008). In goldfish, neurons in the MON respond at the onset of the stimuli or show sustained responses for the duration of the stimuli (Mogdans et al., 1997; Ali et al., 2010; Künzel et al., 2011), and similar responses have been described in the TS (Plachta et al., 1999; Ali et al., 2010).

The algorithm underlying rheotaxis has recently been described theoretically: larvae appear to integrate the difference of flow velocity across their sides to direct turning behavior into the flow of water (Oteiza et al., 2017). This simple decision-making rule aligns them, after a few swim bouts, within the center of the flow velocity gradient. Existing anatomic and physiological descriptions of lateral line processing, described above, are insufficient to explain how this basic vectorial information is encoded in the brain, and how the adaptive decision to turn toward the direction of the flow is generated. Indeed, our current understanding of the brain-wide flow-processing network is insufficient to explain how animals execute a host of behaviors that rely on the lateral line.

In this study, we have used a microfluidic device to deliver water flow stimuli to immobilized zebrafish larvae while imaging a genetically encoded calcium indicator throughout the brain. This has produced whole-brain, cellular-resolution datasets of neural activity in larvae exposed to different speed and orientations of water flow. Our data reveal functional categories of neurons with direction-selective responses to water flow, flow onset, and the accumulation of water flow through time, as well as direction-nonselective neurons responding to water flow or flow onset in either direction. We observe lateral line processing in numerous regions across the brain, including but not limited to those that have been described previously. The results inform a new model of brain-wide water flow processing in larval zebrafish.

\section{Materials and Methods}

Zebrafish larvae and calcium imaging. All procedures were performed with approval from the University of Queensland Animal Welfare Unit in accordance with approval SBMS/378/16/ARC.

Zebrafish (Danio rerio) larvae, of either sex, carrying the transgene elavl3:H2B-GCaMP6s or elavl3:H2B-GCaMP6f (Chen et al., 2013) were maintained at $28.5^{\circ} \mathrm{C}$ on a $14 \mathrm{~h} \mathrm{ON} / 10 \mathrm{~h}$ OFF light cycle. Adult fish were maintained, fed, and mated as previously described in the study by Westerfield (2000). All experiments were conducted in nacre mutant larvae of the TL strain (Chen et al., 2013). Larvae at 6 days postfertilization (dpf) were immobilized in $2 \%$ low-melting point (LMP) agarose (Progen Biosciences) and imaged at $5 \mathrm{~Hz}$ on a custom-built selective plane illumination microscope (SPIM, Thompson et al., 2016; Taylor et al., 2018). The tail was freed below the swim bladder and the fish was paralyzed with $100 \mu \mathrm{M}$ tubocurarine (tubocurarine hydrochloride pentahydrate, Sigma-Aldrich; CHEBI:9774). The flow speed was chosen to be below the startle threshold and was not strong enough to elicit passive movement of the tail. The fish were mounted in a custom-built microfluidics chamber (Fig. 1A) hooked up to a syringe pump (NE-1000X, New Era Pump Systems) that drove water flow, and the flow was triggered using an Arduino board to synchronize with the imaging. The microscope setup was isolated from vibrations on a micro-g lab table (catalog \#63-534, TMC). In each larva, horizontal planes were imaged in either the dorsoventral or ventrodorsal direction, at $20 \mu \mathrm{m}$ increments from the dorsal-most neurons in the brain to the deepest brain region that could be clearly imaged using SPIM. For most larvae, this resulted in a stack of images spanning $\sim 240 \mu \mathrm{m}$ dorsoventrally and capturing the entire rostrocaudal and lateral extents of the brain. This means that most of the brain was robustly sampled, but that some of the deepest regions (composing the ventral-most $50 \mu \mathrm{m}$, approximately) may have been missed in some larvae and may therefore be underrepresented in our dataset. All image acquisition and stimulus presentation was controlled by the microManager software (Edelstein et al., 2010).

Motion artifacts caused by slow drift of the image or by spontaneous movements by the larva were corrected in Fiji (RRID:SCR_002285) using a rigid body transformation in StackReg (Thévenaz et al., 1998). We used the CaImAn toolbox for the fluorescence signal extraction, using 4000 components for the initialization (Pnevmatikakis et al., 2016).

Behavioral experiments. The larvae were mounted as described above, but the imaging was with a $4 \times 0.1$ numerical aperture Olympus microscope objective (PLN $4 \times$ ) placed below the microfluidic device, and a tube lens L4 projected onto a Basler aca1920 camera recording movements at 100 frames per second (fps; Favre-Bulle et al., 2017). Videos were processed using a median filter of 1 pixel radius and background subtraction in Fiji, the tail motion was measured in Fiji and further analyzed in MATLAB (RRID:SCR_001622). The motions were high-pass filtered with a $5 \mathrm{~Hz}$ filter to identify fast tail flicks, and we measured the frequency of tail beats during flow stimulation with the findpeaks function on the high-pass filtered data.

Microparticle imaging velocimetry. Water flow profiles were measured using particle imaging velocimetry (PIV). The flow chamber and pump were set up in a configuration similar to that in imaging experiments, with water replaced by a suspension of $4.5-\mu \mathrm{m}$-diameter polystyrene latex microspheres (Thermo Fisher Scientific) in water at a concentration of $2.5 \times 10^{-4}$ (by weight). The chamber was imaged at $100 \mathrm{fps}$, and the flow field was calculated from 800 sequential images recorded while the pump generated a flow of $10 \mu \mathrm{l} / \mathrm{s}$ (Fig. 1B). The images were analyzed using the PIVlab software package version 2.00 (Thielicke and Stamhuis, 2014).

Clustering and registration of calcium responses. Linear regression, clustering, location, and quantitative analyses were performed in MATLAB, using custom scripts, which can be made available on request. For the first set of experiments, we built regressors, with an average GCaMP response, for each repeat of the flow stimulus onset and offset. The coefficient of determination $\left(r^{2}\right)$ of the linear regression models was used to select responsive neurons, and we chose a 0.1 threshold based on the $r^{2}$ distribution of our models to allow for conservative filtering of the data.

Direction selectivity index (DSI) was calculated as in the study by Grama and Engert (2012).

$$
D S I=\frac{\left(\operatorname{Res}_{h-t}-\operatorname{Res} p_{t-h}\right)}{\left(\operatorname{Res}_{h-t}+\operatorname{Res} p_{t-h}\right)}
$$

Resp $_{\mathrm{h}-\mathrm{t}}$ is the response ( $z$-scored) to head-to-tail (h-t) flow and Resp $\mathrm{t}_{\mathrm{h}}$ is the response to tail-to-head (t-h) flow.

The coordinates of individual neurons were normalized by creating a template from all imaged larvae using ANTs (Advanced Normalization Tools; Avants et al., 2010, 2011). The resulting template was then 

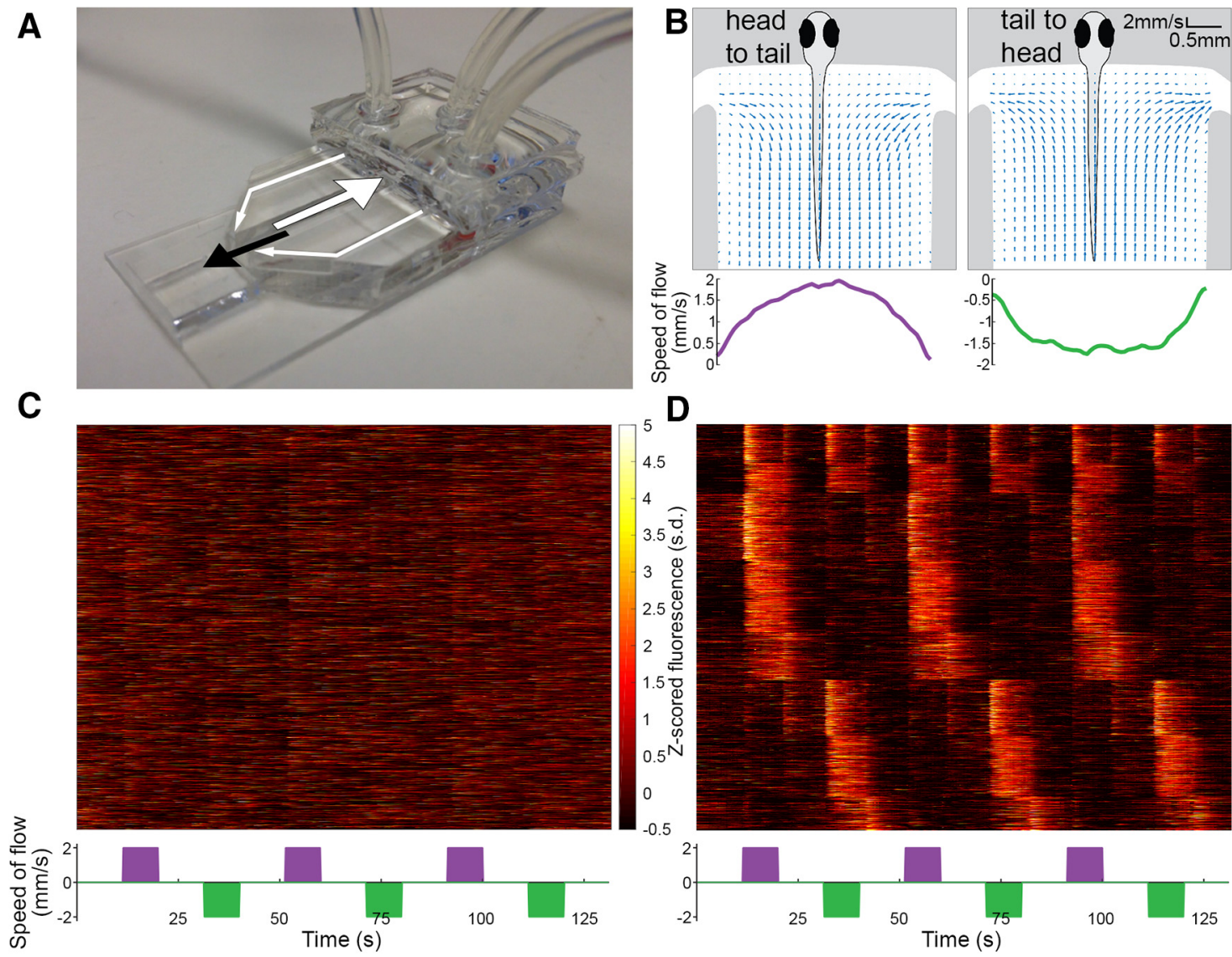

Figure 1. A microfluidics device for presenting larval zebrafish with water flow. A, 3D-printed device, with one large channel and two small channels through which water flows. The flow of water through the channels (for an $\mathrm{h}$-t flow stimulus) is indicated by arrows, and the position and orientation of the larva is indicated with a black arrow. $\boldsymbol{B}$, Micro-PIV analysis of the water flow in the chamber, fish is for illustration only. The traces (bottom) indicate the speed of the flow along the width of the channel. C, Raster plot of the 65,000 fluorescent traces extracted from the 13 fish (left), the direction and timing of the flow is indicated by the colored bars at the bottom (colors match B). $\boldsymbol{D}$, The raster plots for 6510 flow responsive neurons (after $r^{2}$ filtering), grouped by similarity to one another.

registered onto the Z-brain atlas (Marquart et al., 2017), as previously described (Favre-Bulle et al., 2018).

To generate the nodes for the network analysis, the region of interest (ROI) coordinates of each functional cluster were clustered using k-means with a squared Euclidean distance in an unsupervised manner. The number of spatial clusters was constrained by the necessity to have at least 10 ROIs from three different fish contributing to each node; otherwise, the number of spatial clusters was lowered until such a criterion was met. As a result of the light sheet coming from the right side of the fish, we observe a slight asymmetry in the distribution of the nodes, this is explained by the slightly better image quality (signal-to-noise ratio and out-of-focus light). We used the average $z$-scored response of each node to compute a pairwise correlation matrix, and this matrix gave us the correlation metric between each pair of nodes. From this metric, we computed an undirected binary graph where an edge would link two nodes if the correlation between them was above 0.6. Graph metrics were extracted using the brain connectivity toolbox (RRID:SCR_004841; Rubinov and Sporns, 2010). Specifically, we computed the degree (number of edges from each node) and participation coefficient (proportion of different functional clusters that each node is connected to) of each node belonging to a functional cluster and averaged them.

For the assessment of neural decoding of features, we used the Neural Decoding toolbox (https://github.com/KordingLab/Neural_ Decoding/) with linear (Wiener filter), nonlinear (Wiener Cascade), and a dense neural network models (Glaser et al., 2017). We trained each model on the first third, tested on the second third, and validated on the last part of the integrators neuronal responses to the complex flow. We report the Pearson's correlation between the features predicted by each model and the actual features (speed, time, and distance) of the complex flow. To obtain replicates, we split the neuronal population into 10 random groups that were processed in the same way.

Experimental design and statistical analysis. The design included 13 fish for the control group, and 3 fish for the neomycin-treated group in the first set of experiments, as well as 7 fish for the second set of experiments. Linear regressions were performed using the fitlm function of MATLAB with default parameters. The k-means clustering was performed on the filtered data (as described above, $r^{2}>0.1$ ) with 50 clusters using the city block distance with five replicates.

We used a Kolmogorov-Smirnov test in MATLAB to compare the directional selectivity of each cluster against a normal distribution with the following parameters, $\mu$ of 0 (no selectivity) and $\sigma$ of 0.2 corresponding to the mean $\sigma$ of the experimental distributions.

We used an ANOVA (after testing for normality using AndersonDarling tests) in GraphPad prism (RRID:SCR_002798) to compare the mean degrees and participation coefficients. The parameters were set to use the Geisser-Greenhouse correction for nonsphericity, assuming matched measures for each functional clusters and using the Tukey correction for multiple comparisons. We report the adjusted $p$ value, as well as mean differences and mean SE of the differences.

We used Friedman tests in GraphPad Prism to compare the correlation and movement frequencies between baseline and stimuli. We used Dunn's correction for the comparison between areas under the curve and report the adjusted $p$ values.

For the neural decoding, we used a Wilcoxon test to compare the correlation between time and distance in our pseudo-replicates, and we report the one-tailed $p$ value. 


\section{Results}

Combining microfluidics and light sheet microscopy to detect water flow responses

Brain-wide mapping of water flow networks requires the controlled application of flow stimuli in a setting where calcium imaging can take place. With this goal, we designed a 3D-printed microfluidic device compatible with our custom-built light sheet microscope (Fig. 1A; Favre-Bulle et al., 2018; Taylor et al., 2018). We embedded 6 dpf larvae in LMP agarose to immobilize them, cut the agarose away from their tails below the swim bladder, and inserted them into the device's larger channel. We then used a dual-syringe pump to deliver smooth consistent stimuli, pushing water through the small side channels and pulling it out of the center channel to produce h-t flow, or reversing this to produce $\mathrm{t}$-h flow. To gauge the resulting water flow stimuli, we suspended polystyrene latex beads in water and imaged their movements while the pump was active (Fig. 1B). This preparation produced an orderly flow that peaked at $2 \mathrm{~mm} / \mathrm{s}$ in the middle of the chamber where the tail of the fish was positioned.

We first performed a broad unbiased search for water flowresponsive neurons across the brain. We started with a simple stimulus train of alternating h-t and $\mathrm{t}-\mathrm{h}$ flow (10 s each, with $10 \mathrm{~s}$ of rest between stimuli) with a single consistent flow rate of 2 $\mathrm{mm} / \mathrm{s}$, well below the threshold for eliciting startles and within the bounds of larval swim speed (McHenry et al., 2009; Severi et al., 2014). Thirteen fish expressing a pan-neuronal nuclear-targeted GCaMP6s (Chen et al., 2013) were paralyzed with tubocurarine and exposed to the above stimulus in the microfluidics device, while whole-brain calcium imaging proceeded. Following motion correction and segmentation of our images into ROIs corresponding to neurons (see Materials and Methods), we obtained fluorescence traces for $\sim 63,000$ ROIs (Fig. $1 C$ ). We registered these ROIs to the Z-brain atlas, a reference atlas for $6 \mathrm{dpf}$ larval zebrafish in which brain regions were identified through both anatomic location and expression patterns, allowing responses to be registered spatially from animal to animal, and against the brain regions delineated in Z-brain (Randlett et al., 2015). As previously described (Favre-Bulle et al., 2018), we used linear regression to identify $\sim 6500$ ROIs that were consistently and specifically responsive to flow stimuli (Fig. 1D).

\section{A basic characterization of flow-responsive neurons across the larval zebrafish brain}

Our next goal was to categorize the different types of responses that water flow elicits across the brain. From our 6500 water flow-responsive ROIs, we used k-means clustering with a city block distance to identify eight distinct functional clusters of water flow-responsive neuron (Figs. 2, 3). One broad category of flow-responsive ROIs corresponded to onset-specific neurons (Fig. 2A, Movie 1) and comprised the following three functional clusters: "bidirectional onset" neurons that had similar responses to flow in either direction; "h-t onset" neurons responsive selectively responsive to h-t flow; and "t-h onset" neurons specifically responsive to $\mathrm{t}$-h flow. In each case, there was an initially strong response to the stimulus that quickly settled back to baseline, even as the flow stimulus persisted. Each also showed a weak response to the offset of the opposite direction stimulus, or to the cessation of stimuli of both directions in the case of bidirectional onset neurons. This could result from either a small amount or backflow in the chamber at the end of these stimuli or a weak sensitivity to relative changes in flow rate (such that the cessation of $\mathrm{t}$-h flow is equivalent to the onset of h-t flow).
ROIs belonging to the three "onset" clusters were distributed broadly across the brain, and overlapped extensively with one another, especially in the hindbrain. There were, however, differences in the density of different clusters in different brain regions, with bidirectional onset neurons enriched in the TS (Figs. 2A, 4).

A second broad category of ROIs responded to the presence of flow with activity that persisted for the duration of the stimulus (Fig. 2B, Movie 2). These included clusters of "bidirectional on," "h-t on," and "t-h on" neurons, paralleling the direction selectivity shown by the onset neurons described above. Again, the spatial distributions of these three clusters were highly overlapping, especially in the hindbrain and $\mathrm{MON}$, but regional enrichments for specific clusters were also evident (Figs. 2B, 4). Notably, the directional-selective on clusters (of both orientations) were the nearly exclusive occupants of the pLLG, reinforcing the observations that the responses of these neurons reflect activity in the neuromasts (Pichler and Lagnado, 2019).

The final broad category of ROI, comprising the "h-t integrator" and "t-h integrator" clusters, showed persistent responses that grew in intensity throughout the duration of the stimulus, then gradually returned to baseline after the stimulus ended (Fig. $2 C$, Movie 3). Our analysis did not reveal bidirectional integrators. The h-t integrators were more numerous, but the distributions of h-t and $\mathrm{t}$-h integrators were similar, with a majority of neurons in these clusters appearing in the tectum or the hindbrain.

Quantitative analyses of the response properties of these clusters bore out the initial observations presented above. Six of the eight clusters showed strong direction selectivity (according to the DSI; Eq. 1), with only the two bidirectional clusters failing to diverge significantly from 0 (Fig. $2 D$ ). In terms of the time that ROIs took to reach their peak responses to water flow stimuli, the three onset clusters showed fast precise responses, the on clusters had average peaks near the midpoint of the $10 \mathrm{~s}$ stimuli, and the integrator clusters peaked at or just after the termination of the stimulus (Fig. 2E).

Clustering is subject both to the inadvertent separation of equivalently responding ROIs (overclustering) and to the combining of distinctly responding ROIs (underclustering). While it is not possible to exclude the possibility of either of these types of error in our clustering, we performed a t-SNE ( $t$-distributed stochastic neighbor embedding for long) dimension reduction, using the correlation distance and not the city block distance of our k-means, to visualize the members of the eight clusters (Fig. $2 F)$ relative to one another. Reassuringly, the eight clusters show clear segregation, with the h-t- and $\mathrm{t}$-h-selective clusters separated, and the bidirectional clusters in between the two. Within each of these direction-selective groups, the subclasses of functional responses are also separated. These observations provide support for the validity of our eight clusters as biologically relevant subtypes of flow-responsive neuron.

As a final control to confirm that we are observing bona fide lateral line responses, we treated larvae with neomycin to ablate their lateral line hair cells (Harris et al., 2003). Following lateral line ablation, we observed a drastic drop in flow responsiveness across the brain, as judged by the distribution of the coefficient of determination of all ROIs to the same simple stimulus train used above (Fig. 5A). In attempting to cluster the responses that did occur, we found very small numbers of neurons fitting into two noisy clusters approximately corresponding to the h-t on and $\mathrm{t}-\mathrm{h}$ on clusters from our initial experiments, albeit with weaker response strength (Fig. 5). The small number of responsive ROIs, along with their poor response fidelity, indicates that 


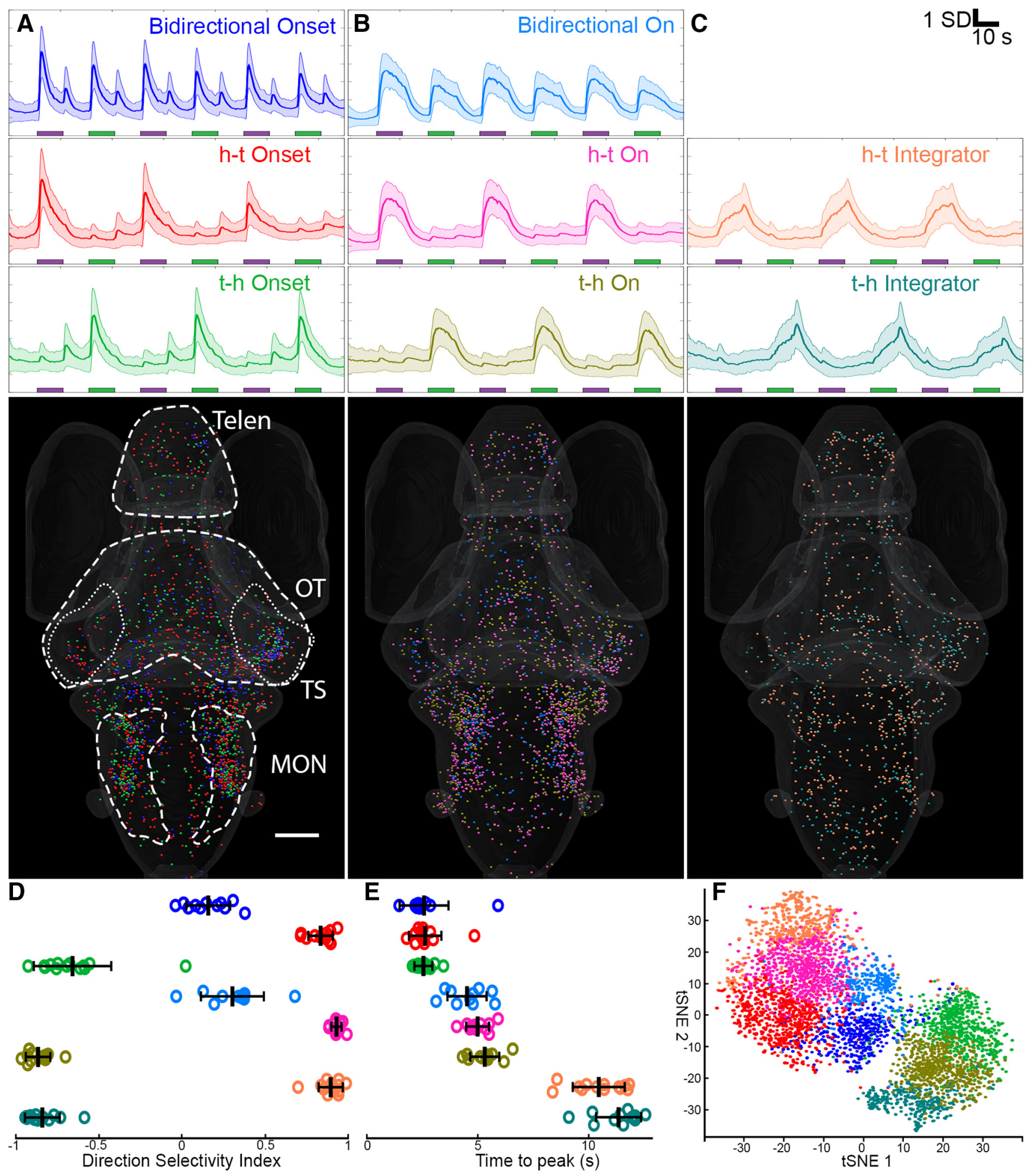

Figure 2. Whole-brain response profiles to the water flow stimuli. $\boldsymbol{A}$, The first group of responses show a rapid response to the onset of the stimuli, indicated by colored rectangles (mean responses, SD shaded area). Some responses are weakly direction selective (top), the others are selective to h-t (middle) or t-h (bottom) water flow. Outlines indicate telencephalon (Telen), 0T, TS (dotted), and MON. Scale bar, $100 \mu \mathrm{m}$. Rotation in Multimedia 1. B. The second group shows ongoing responses for the duration of the stimuli (mean responses, SD shaded area). These can again be subdivided by their direction selectivity: weakly h-t (top), strongly h-t (middle), or strongly t-h (bottom). Rotation in Multimedia 2. C, The third group shows a slow buildup of the GCaMP signal, peaking at the end of the stimuli. Only two such clusters fit into this category: one strongly selective for h-t flow and the other strongly selective for $\mathrm{t}-\mathrm{h}$ flow. Rotation in Multimedia 3. D, Scatter plot of the average DSI of each cluster. DSI $=0.16 \pm 0.13,0.83 \pm 0.07,-0.66 \pm 0.23,0.30 \pm 0.19,0.93 \pm 0.03,-0.87 \pm 0.07,0.9 \pm 0.07,-0.84 \pm 0.1$; mean $\pm S D, n=13$ fish). The $p$ values of KS tests against normal distribution $(\mu=0, \sigma=0.2)=2.4981 \mathrm{e}-02,1.8228 \mathrm{e}-04,5.9128 \mathrm{e}-12,5.8589 \mathrm{e}-12,7.6683 \mathrm{e}-13,5.9769 \mathrm{e}-11,5.9260 \mathrm{e}-12$, 6.3809e-12. $E$, Scatter plot of the average time to maximum intensity of each cluster, both for the h-t (left) and t-h (right) stimuli $(2.3 \pm 1.1,4.3 \pm 0.9,2.4 \pm 0.7,4.7 \pm 0.5,10.2 \pm 1.2$, $13.7 \pm 3.4,13.7 \pm 4.6,8.3 \pm 5.6$; mean $\pm S D, n=13$ fish). $\boldsymbol{F}$, tSNE plot of all the members from the eight clusters, using correlation as a distance. 3-dimensional rotations of the dorsal views shown in $\boldsymbol{A}, \boldsymbol{B}$, and $\boldsymbol{C}$ can be found in Movies 1, 2, and 3, respectively. 


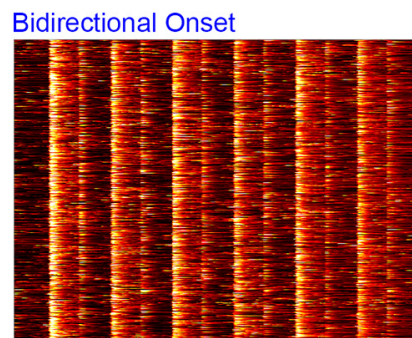

Bidirectional On
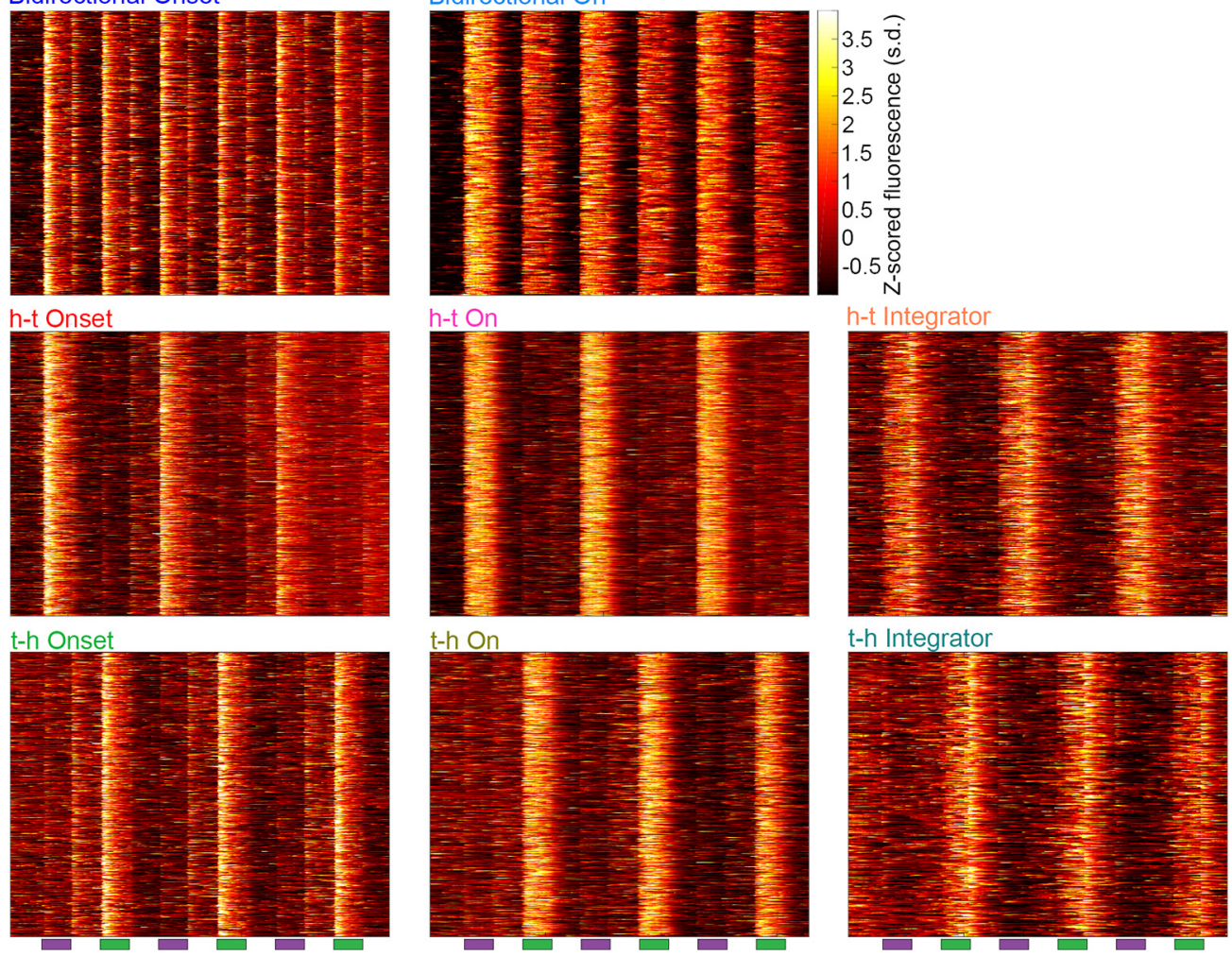

t-h Integrator

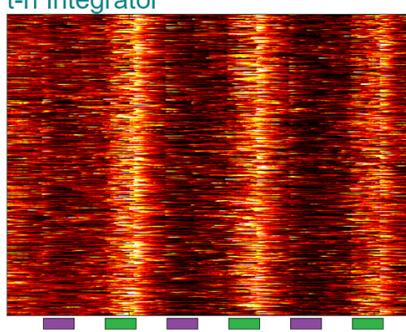

Figure 3. Heat maps of the eight clusters of flow-responsive neurons. The $z$-scored fluorescent traces of all the ROls composing each of the eight clusters from Figure 2. Colored rectangles at the bottom indicate the timing of the stimuli (magenta for h-t, green for t-h).

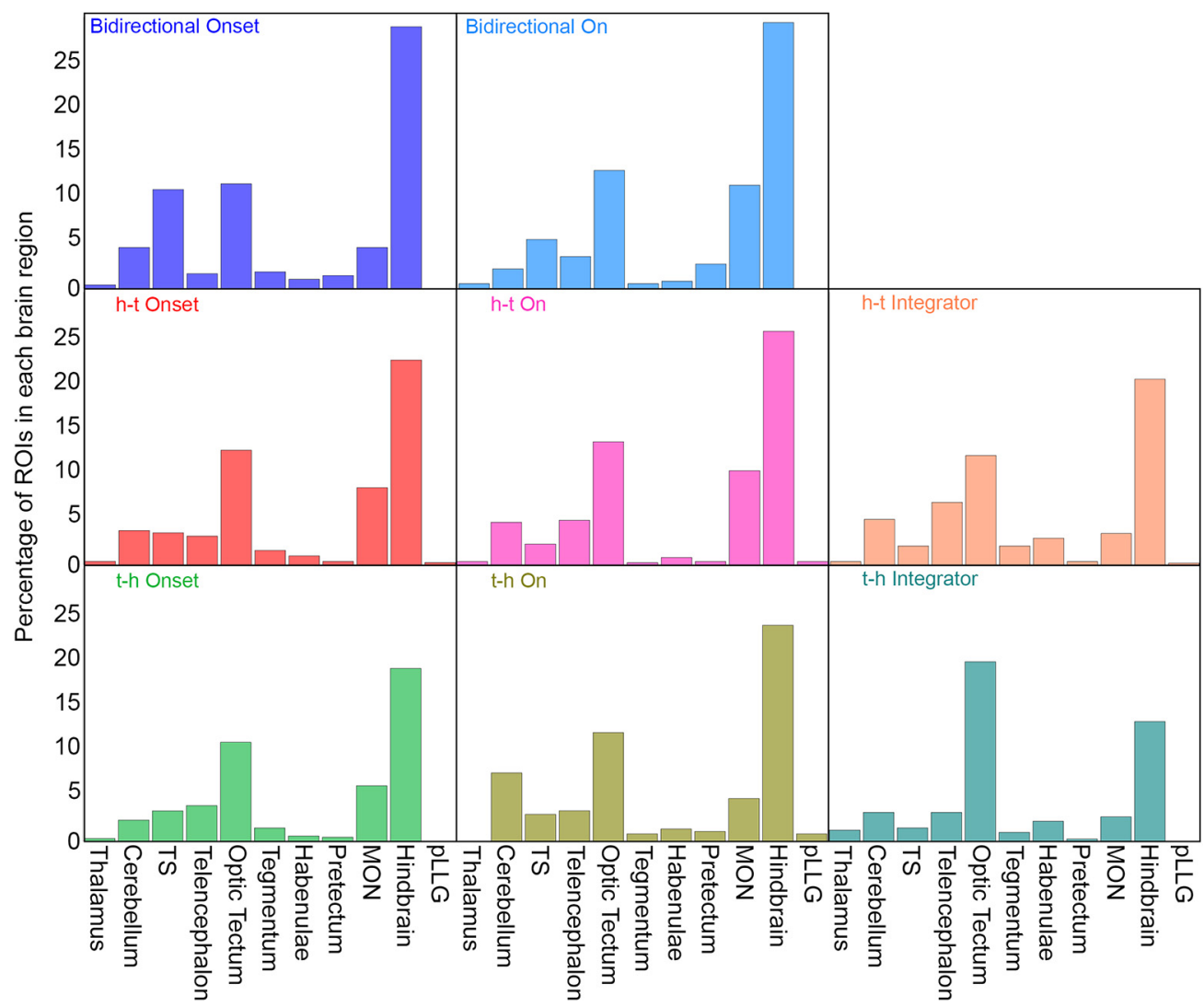

Figure 4. Distribution of the eight clusters across flow-responsive brain regions. Normalized distribution of each cluster from Figure 2 across the major flow-responsive brain regions. 
A

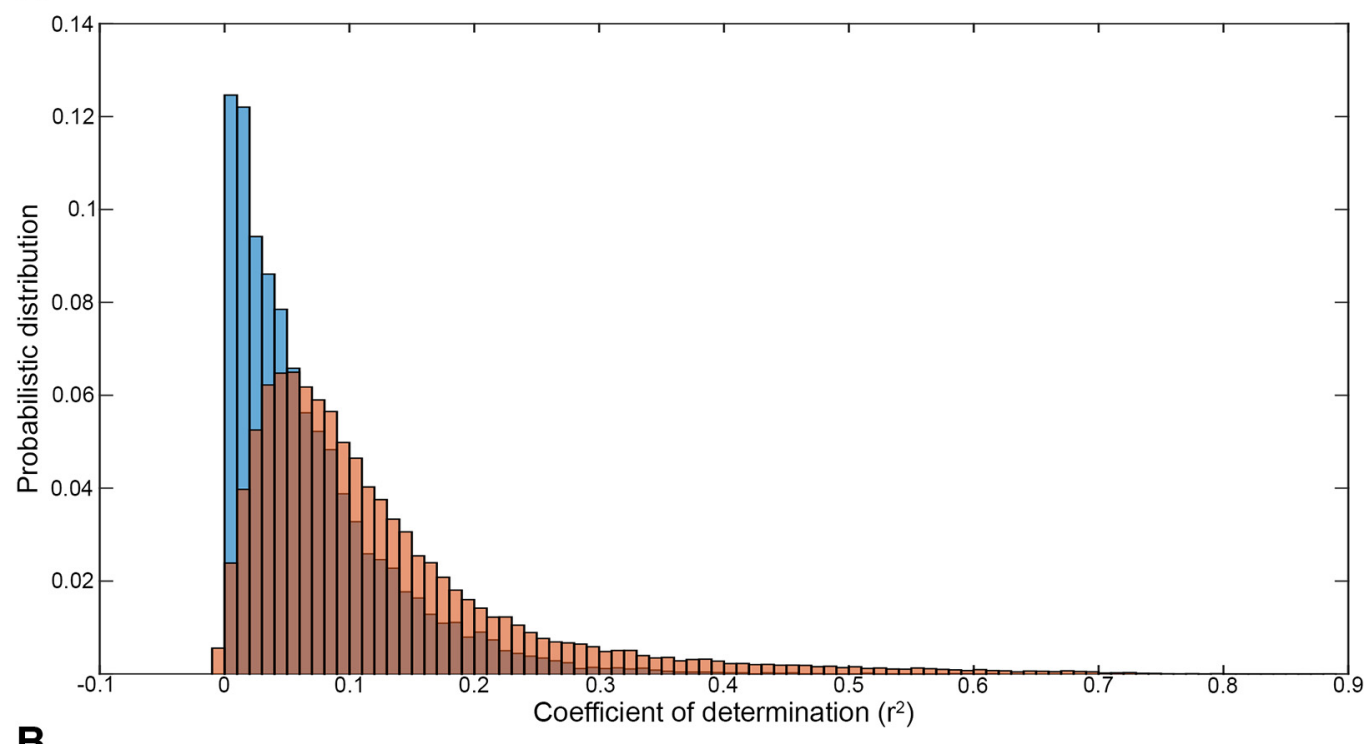

B
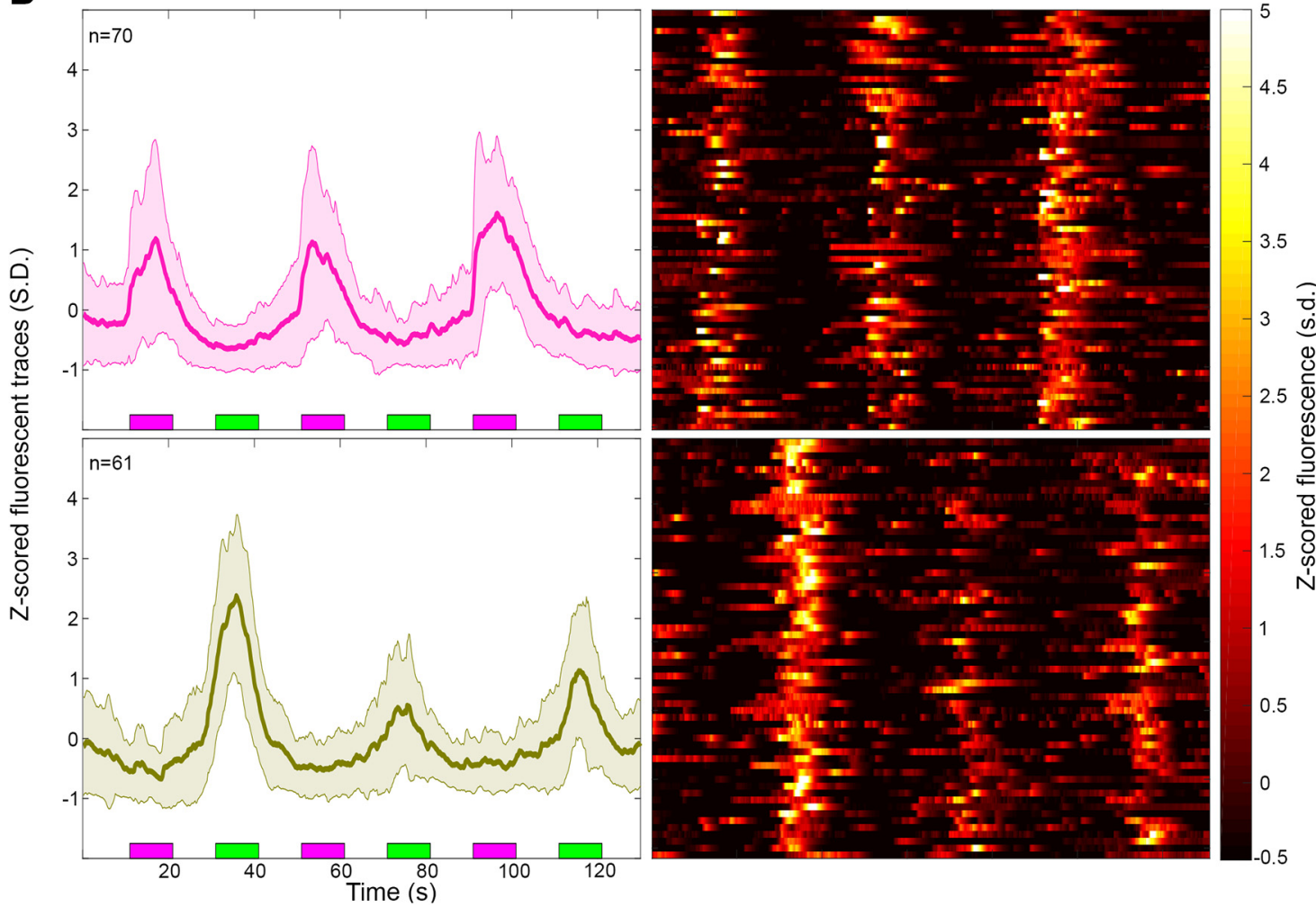

Figure 5. Neomycin-treated whole-brain responses to water flow. $A$, Normalized distribution of the $r^{2}$ values from the linear regression of each fluorescence trace for every R0l to the water flow stimuli, WT in orange and neomycin-treated, with their lateral line ablated, fish in blue $(n=3)$. $\boldsymbol{B}$, Clustered responses, after filtering on the $r^{2}$ values as we did in Figure 2 from the neomycin-treated fish, average responses (left), and heatmaps (right). Colored rectangles at the bottom indicate the timing of the stimuli (magenta for $h-t$, green for t-h).

the neomycin is blocking $\sim 90 \%$ of the flow responses. This suggests that the lateral line system rather than, for instance, proprioception, is responsible for the numerous, strong, and diverse responses that we describe above.

\section{Correlation patterns across the brain-wide water flow network}

To gain a better sense of the network organization of the observed responses, we applied the tools of graph theory to our results. We performed an unsupervised clustering of the spatial localization of all the responsive ROIs, for each functional cluster, ensuring that the nodes would be represented in all fish (Fig. 6D). This unsupervised approach confirmed the prevalence of mostly h-t and $t-h$ on clusters in the pLLG (Fig. 6D, dashed outlines). We then generated an average correlation matrix between all the nodes across our fish, with correlation $<0.6$ ignored, to generate an undirected graph (Fig. $6 A, E)$. The graph metrics of degree and participation across clusters were consistent at other thresholds of correlation, which shows that the 0.6 correlation threshold is not special and the following results are generalizable independent of the threshold (Fig. 6C). A null model was 

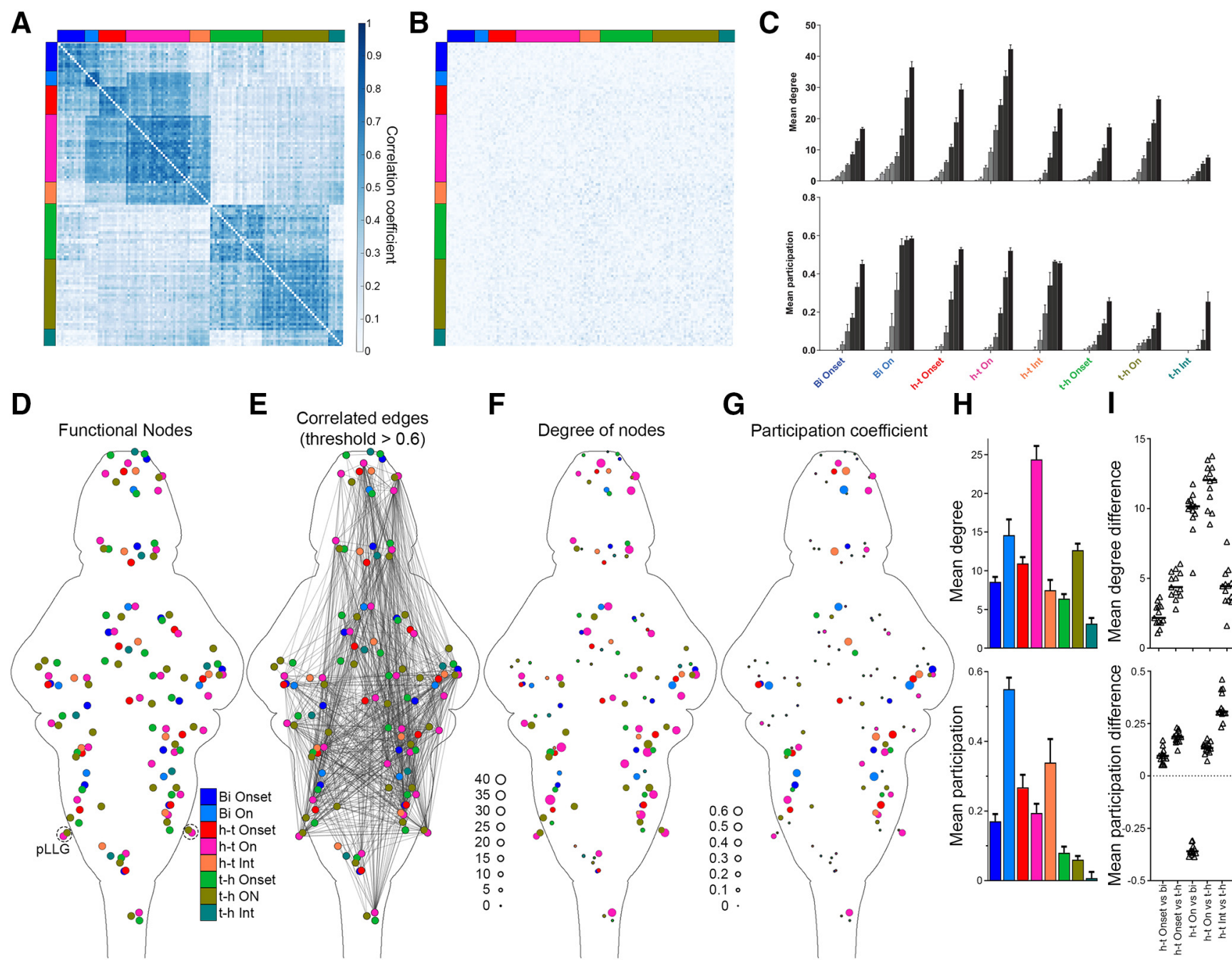

G Participation coefficient H

Figure 6. Graph analysis of flow-responsive network. $\boldsymbol{A}$, Correlation matrix for the 126 functional nodes. Color bar indicates correlation coefficient. $\boldsymbol{B}$, Amplitude adjusted Fourier transform null model correlation matrix. $\boldsymbol{C}$, We computed the degree and participation of the graph from Figure 3 using lowering correlation thresholds from 0.9 (light gray) to 0.5 (black). D, Spatial localization of the representative 126 nodes from our eight functional clusters, colored as in Figure 2 . The light sheet enters the brain from the right, which gives us as better signal on that side and explains the observed assymetry. $\boldsymbol{E}$, Graph showing functional edges between nodes with correlation coefficients exceeding 0.6. $\boldsymbol{F}$, The sizes of the nodes represent the degree (number of connections) of each node. Larger nodes have more connections. $\boldsymbol{G}$, Participation coefficient between functional classes (fraction of different classes with which each node is connected), with larger nodes having stronger participation scores. $\boldsymbol{H}$, Bar plots of mean degree and mean participation ( $\pm S D, n=13$ fish) across all nodes from each of the eight functional cluster. $p$ Values and mean differences for the multiple comparisons can be found in Tables 1 and 2 for degrees and participation, respectively. $\boldsymbol{I}$, Scatter plots of the difference of mean degree and mean participation from $\boldsymbol{H}$, for each fish, comparing the h-t clusters to their equivalent clusters in other directions.

generated by applying an amplitude-adjusted Fourier transform to the average time series of each node, which preserves the amplitude and temporal structure of the data while shuffling the temporal indices (Fig. 6B; Theiler et al., 1992). This null model loses any structure and shows no correlation higher than our threshold (highest correlation, 0.33 ).

We observe that the network is biased toward the h-t on response cluster, which is significantly more connected as measured by the degree of each node (the number of nodes to which each node, regardless of function, is correlated; Fig. $6 F, H, I$, Table 1). We also compared the participation coefficient (the proportion of different functional clusters with which each node connects) and again the h-t-specific clusters had a significantly higher participation coefficient than t-hspecific ones (Fig. 6G,I, Table 2). Overall, this suggests a network that encodes various properties of flow stimuli in both directions, but with a greater network-wide weighting on the detection and processing of h-t flow.
Flow-responsive neurons encode specific properties of water flow stimuli

Our identification of "on," "onset," and "integrator" neurons, along with functional clusters within these categories responsive to $\mathrm{h}-\mathrm{t}$ flow, $\mathrm{t}-\mathrm{h}$ flow, or both reveals the cardinal response types to flow information in the larval zebrafish brain. Our simple stimulus train, however, limits our ability to gauge how these neurons encode key properties of flow stimuli, including their speed, duration, and overall magnitude. Specific questions include whether any of these neurons encode speed, whether onset neurons respond only to the onset of stimuli or to changes in speed while a stimulus is ongoing, and whether integrator neurons accumulate the duration or volume of flow. To explore these response properties further, we designed a more complex stimulus train (Fig. $7 A$, top) with stimuli of various strengths, directions, and durations, and we paired or separated these stimuli temporally to isolate responses to stimulus onset, change in flow speed, and stimulus offset. To increase the temporal 
Table 1. Multiple comparison of mean degree of nodes from Figure 3

\begin{tabular}{|c|c|c|c|}
\hline Tukey's multiple-comparisons test & Mean differences & SE of differences & Adjusted $p$ values \\
\hline $\mathrm{Bi}$ vs $\mathrm{Bi}$ On & -6.026 & 0.4368 & $2.03924 \mathrm{E}-07$ \\
\hline Bi vs h-t Onset & -2.365 & 0.2208 & $3.41308 \mathrm{E}-06$ \\
\hline Bi vs h-t On & -15.81 & 0.4668 & $4.602 \mathrm{E}-12$ \\
\hline Bi vs h-t Int & 1.068 & 0.3621 & 0.145276416 \\
\hline Bi vs t-h Onset & 2.179 & 0.2924 & 0.000147338 \\
\hline Bi vs t-h On & -4.08 & 0.3237 & $5.68213 \mathrm{E}-07$ \\
\hline Bi vs t-h Int & 5.399 & 0.3284 & $2.78814 \mathrm{E}-08$ \\
\hline Bi vs h-t Onset & 3.66 & 0.4634 & $8.29456 \mathrm{E}-05$ \\
\hline Bi vs h-t On & -9.784 & 0.4253 & $5.56424 \mathrm{E}-10$ \\
\hline Bi vs h-t Int & 7.094 & 0.543 & 3.79913E-07 \\
\hline Bi vs t-h Onset & 8.205 & 0.6078 & 2.61099E-07 \\
\hline Bi vs t-h On & 1.945 & 0.6091 & 0.099411707 \\
\hline Bi vs t-h Int & 11.42 & 0.6618 & $1.65661 \mathrm{E}-08$ \\
\hline h-t vs h-t On & -13.44 & 0.3651 & $2.775 \mathrm{E}-12$ \\
\hline h-t vs h-t Int & 3.434 & 0.2691 & 4.96249E-07 \\
\hline h-t vs t-h Onset & 4.545 & 0.2686 & $2.04588 \mathrm{E}-08$ \\
\hline h-t vs t-h On & -1.715 & 0.2511 & 0.000342965 \\
\hline$h-t$ vs t-h Int & 7.765 & 0.3452 & 7.68203E-10 \\
\hline h-t vs h-t Int & 16.88 & 0.3431 & $2.01 \mathrm{E}-13$ \\
\hline h-t vs t-h Onset & 17.99 & 0.4323 & $1.099 \mathrm{E}-12$ \\
\hline h-t vs t-h On & 11.73 & 0.423 & $2.7792 \mathrm{E}-11$ \\
\hline h-t vs t-h Int & 21.21 & 0.5021 & $9.64 \mathrm{E}-13$ \\
\hline h-t vs t-h Onset & 1.111 & 0.3568 & 0.112628911 \\
\hline h-t vs t-h 0n & -5.149 & 0.3091 & $2.42072 \mathrm{E}-08$ \\
\hline$h-t$ vs t-h Int & 4.331 & 0.394 & $2.58489 \mathrm{E}-06$ \\
\hline$t-h$ vs $t-h 0 n$ & -6.26 & 0.1799 & $3.941 \mathrm{E}-12$ \\
\hline$t-h$ vs $t-h$ Int & 3.22 & 0.1841 & $1.44663 \mathrm{E}-08$ \\
\hline$t-h$ vs $t-h$ Int & 9.48 & 0.2121 & $5.65 \mathrm{E}-13$ \\
\hline
\end{tabular}

$\mathrm{Bi}$, Bidirectional; Int, integrator.

resolution of our calcium imaging during these rapid transitions, we used GCaMP6f, a GCaMP variant with faster kinetics than the GCaMP6s used above (Chen et al., 2013). This will also allow us to validate that some of the response profiles were not artifacts of the slow rise and decay of GCaMP6s.

We first used the eight previous functional clusters from our simple $\mathrm{h}-\mathrm{t} / \mathrm{t}-\mathrm{h}$ stimulus train to predict responses to our more complex stimulus train (Fig. $7 A$, top), we obtained ROIs with similar profiles across our seven fish (Fig. $7 A$, raster plot). However, we failed to identify responses corresponding to the $\mathrm{t}$-h integrators, which may indicate that these were an artifact of the GCaMP6s, of our clustering approach, or both.

Using the response profiles to this more complex stimulus train (Fig. $7 A$, top), we asked specific questions regarding the salient stimulus features responsible for activity in the onset neurons. Are they triggered specifically at the start of a flow stimulus, or are they sensitive to any changes in flow speed? To address this, our complex stimulus train included a $30 \mathrm{~s}$ stimulus that changed from 1 to $2 \mathrm{~mm} / \mathrm{s}$ after $10 \mathrm{~s}$, stayed at $2 \mathrm{~mm} / \mathrm{s}$ for $10 \mathrm{~s}$, and then dropped back to $1 \mathrm{~mm} / \mathrm{s}$ for the final $10 \mathrm{~s}$. Clustering of the onset responses for a restricted window of time surrounding these changes of speed did not reveal consistent responses to the change from 1 to $2 \mathrm{~mm} / \mathrm{s}$, indicating that the onset cells represent the start of flow, but not a change of speed (Fig. $7 B$ ).

Our next question involved the integrators and which of the following flow features they integrate: time, or a combination of time and speed, which would be equivalent to the volume of water flow experienced by the fish. As mentioned above, we failed to identify the $t-h$ integrator responses, but the h-t integrator showed responses that correlated strongly to a combination of the time and speed of the flow, which may represent the amount of fluid displacement of the flow stimulus, or simulated motion from the fish frame of reference (Fig. 7C, Movie 4).
Table 2. Multiple comparison of mean participation of nodes from Figure 3

\begin{tabular}{|c|c|c|c|}
\hline Tukey's multiple-comparisons test & Mean differences & SE of differences & Adjusted $p$ values \\
\hline Bi vs $\mathrm{Bi}$ On & -0.3795 & 0.006901 & $6 \mathrm{E}-14$ \\
\hline Bi vs h-t Onset & -0.09528 & 0.009757 & 9.22024E-06 \\
\hline Bi vs h-t On & -0.02384 & 0.005464 & 0.014718504 \\
\hline Bi vs h-t Int & -0.1690 & 0.01847 & $1.83232 \mathrm{E}-05$ \\
\hline Bi vs t-h Onset & 0.09016 & 0.005358 & 2.17055E-08 \\
\hline Bi vs t-h On & 0.1103 & 0.006055 & $9.453 \mathrm{E}-09$ \\
\hline Bi vs t-h Int & 0.1630 & 0.006445 & $1.24898 \mathrm{E}-10$ \\
\hline Bi vs h-t Onset & 0.2842 & 0.01155 & $1.97298 \mathrm{E}-10$ \\
\hline Bi vs h-t On & 0.3556 & 0.006867 & $1.12 \mathrm{E}-13$ \\
\hline Bi vs h-t Int & 0.2105 & 0.02085 & $6.46912 \mathrm{E}-06$ \\
\hline Bi vs t-h Onset & 0.4697 & 0.008355 & $4.9 \mathrm{E}-14$ \\
\hline Bi vs t-h On & 0.4898 & 0.009543 & $1.24 \mathrm{E}-13$ \\
\hline Bi vs t-h Int & 0.5425 & 0.008934 & $3.1 \mathrm{E}-14$ \\
\hline h-t vs h-t On & 0.07144 & 0.008653 & $5.30168 \mathrm{E}-05$ \\
\hline h-t vs h-t Int & -0.07375 & 0.01843 & 0.026659926 \\
\hline h-t vs t-h Onset & 0.1854 & 0.008249 & $7.74868 \mathrm{E}-10$ \\
\hline h-t vs t-h 0n & 0.2056 & 0.01104 & 7.45706E-09 \\
\hline h-t vs t-h Int & 0.2583 & 0.01215 & $1.6358 \mathrm{E}-09$ \\
\hline h-t vs h-t Int & -0.1452 & 0.01616 & 2.21301E-05 \\
\hline h-t vs t-h Onset & 0.1140 & 0.006857 & 2.47249E-08 \\
\hline h-t vs t-h 0n & 0.1341 & 0.007899 & $1.97511 \mathrm{E}-08$ \\
\hline h-t vs t-h Int & 0.1869 & 0.007428 & $1.36734 \mathrm{E}-10$ \\
\hline h-t vs t-h Onset & 0.2592 & 0.01891 & 2.19286E-07 \\
\hline h-t vs t-h 0n & 0.2793 & 0.01875 & $8.41255 \mathrm{E}-08$ \\
\hline h-t vs t-h Int & 0.3321 & 0.01894 & $1.41224 \mathrm{E}-08$ \\
\hline t-h vs t-h On & 0.02011 & 0.003816 & 0.003446007 \\
\hline$t-h$ vs $t-h$ Int & 0.07286 & 0.007618 & 1.15143E-05 \\
\hline$t-h$ vs $t-h$ Int & 0.05275 & 0.006182 & 3.78496E-05 \\
\hline
\end{tabular}

Bi, Bidirectional; Int, integrator.

Specifically, we measured the areas under the response curves during different combinations of flow speed and duration for the h-t flow. This showed that the area under the curve does not appear to increase simply for either time or speed, but that responses from integrators are impacted both by the time and speed of the stimulus (Fig. 7C). We speculate that the lack of significant difference between the $1 \mathrm{~mm} / \mathrm{s}$ for 10 or $20 \mathrm{~s}$ stimulus could be an artifact of increased responses to the first stimulus presented in the stimulus train (the $10 \mathrm{~s}$ stimulus, as also observed for several clusters in Fig. 2A; Favre-Bulle et al., 2018), or could be explained by a floor effect. To validate which feature the integrators may best encode, we used the Neural Decoding toolbox (Glaser et al., 2017) and trained three models on the integrators neuronal responses (see Table 5). In all three models, the distance feature (combining speed and duration) best explained by the responses, ahead of either speed or duration individually. The difference between time and distance encoding was significant in all three models [Wiener filter (linear), $p=0.002$; Wiener cascade (nonlinear), $p=0.002$; and dense neural network, $p=0.001$; one-tailed Wilcoxon test).

In larval zebrafish, different water flow speeds trigger different behaviors ranging from rheotaxis to startles (McHenry et al., 2009; Olszewski et al., 2012; Suli et al., 2012; Oteiza et al., 2017), suggesting that the speed of flow stimuli is represented in the brain. To search for speed-encoding ROIs, we observed responses that occurred during the pooled stimuli at 1 or $2 \mathrm{~mm} / \mathrm{s}$ of flow in our complex stimulus train. In analyzing the resulting data, we identified putative speed encoding ROIs by selecting all responses that differed by $>2$ SDs between the two speeds, and then clustered these ROIs to separate noisy or inconsistent responses from those possibly encoding speed. This targeted analysis yielded two functional clusters (Fig. 7D, Movie 5), one with approximately threefold stronger responses to flow at 2 than $1 \mathrm{~mm} / \mathrm{s}$ (680 ROIs 

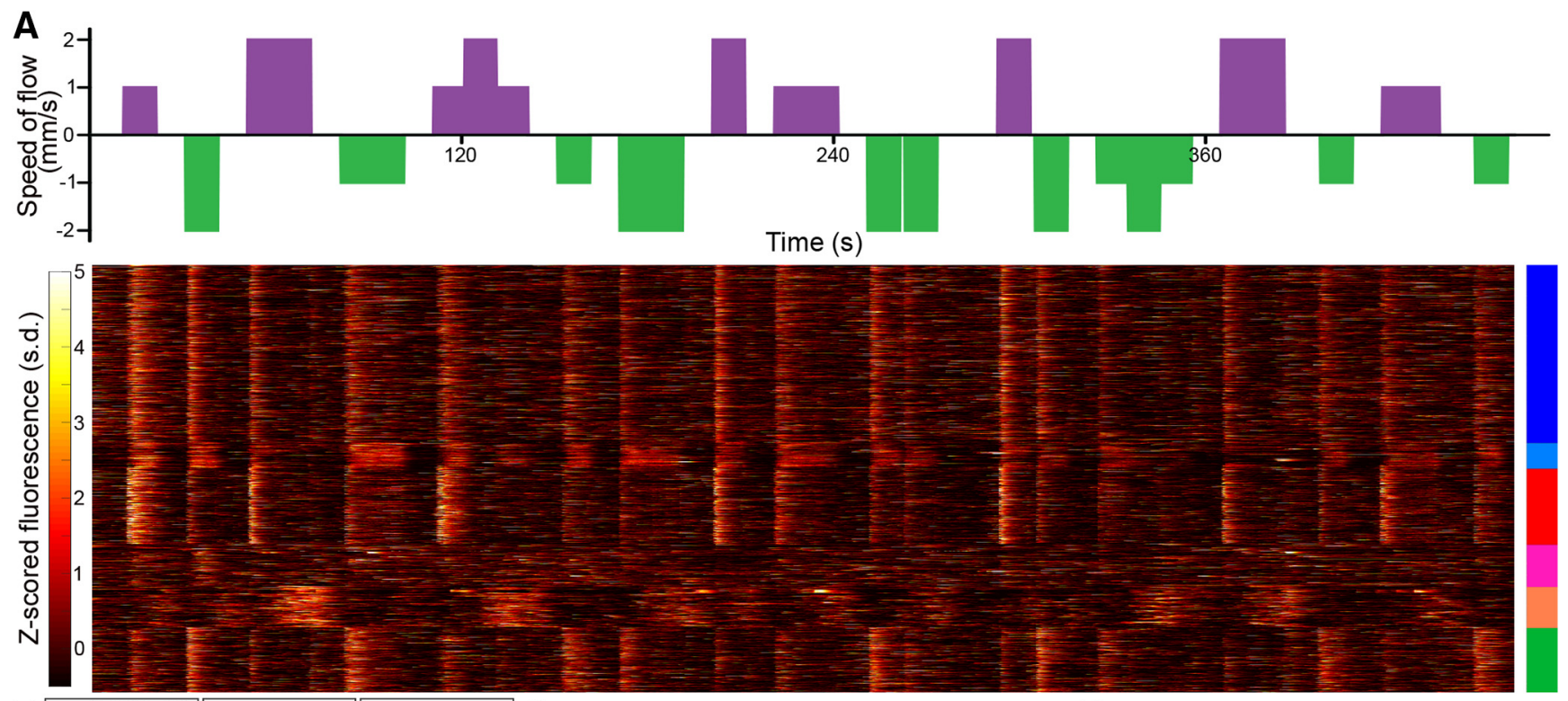

B
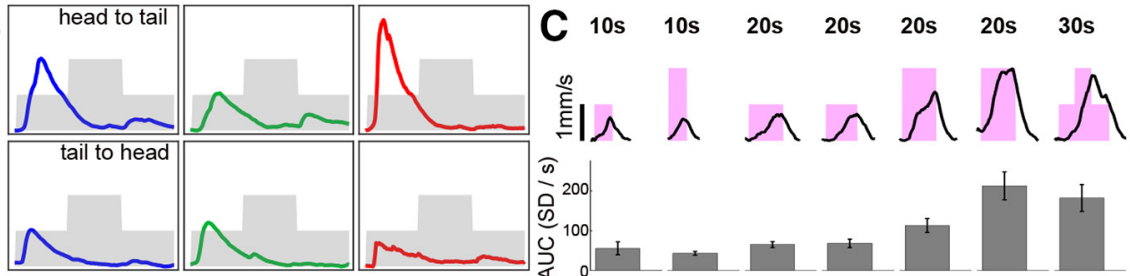

D
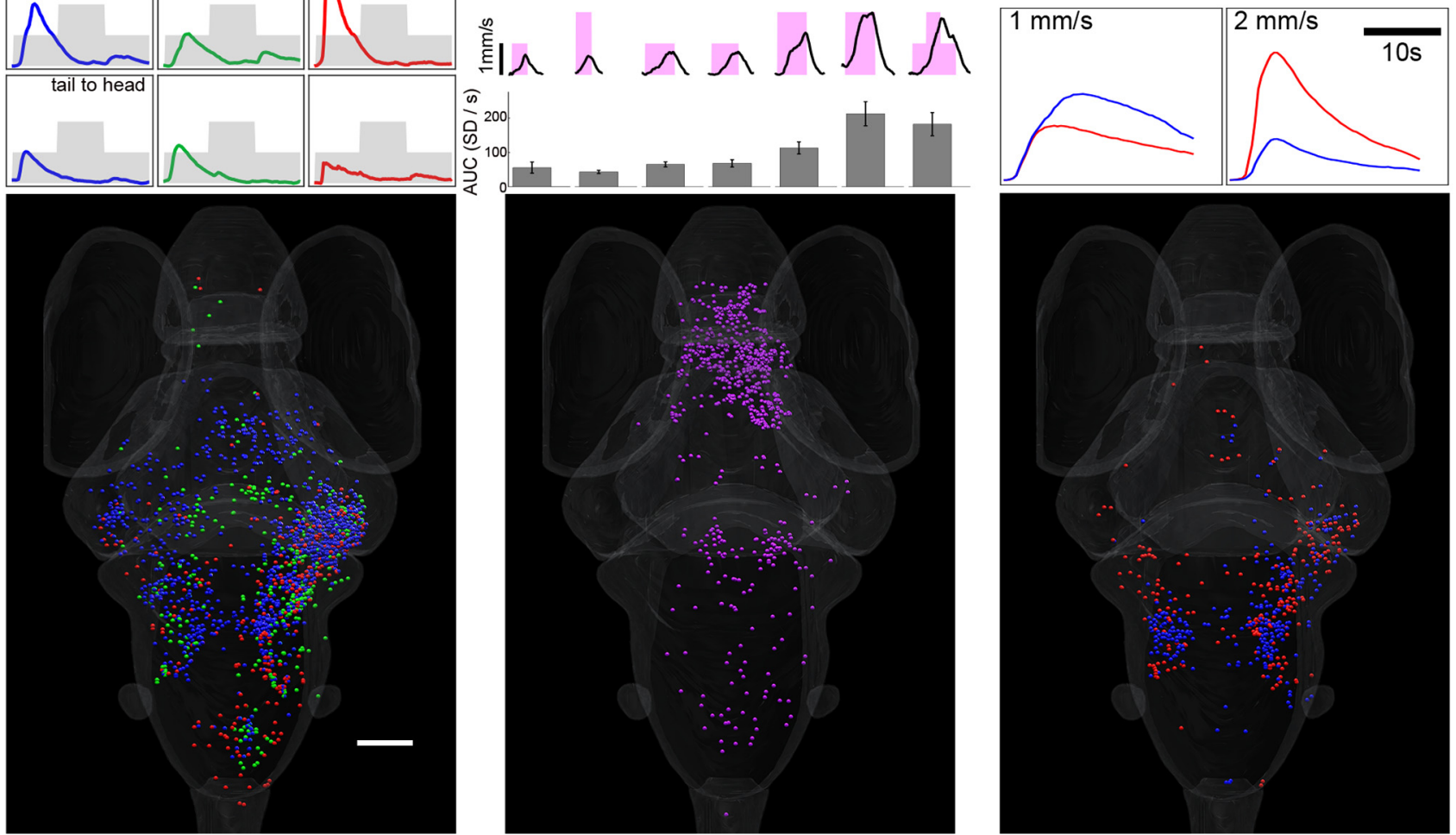

Figure 7. Brain-wide representations of the complex features of water flow. A, A more complex stimulus train was used to assess the representation of speed, acceleration, and volume through the whole brain. At the top is a visual representation of the stimulus train (forward simulated motion, or $\mathrm{h}$-t flow, is positive in magenta; t-h flow is negative in green). The bottom shows the raster plot of the responses similar to the clusters of the simple stimulus from Figure 2. Colored rectangles indicate to which functional clusters from Figure 2 the ROls belong to. $\boldsymbol{B}$, Onset functional clusters do not respond to stepwise change in speed. Top, Average response for the bidirectional, t-h-specific, and h-t specific onset clusters. Bottom, Visualization of their localization (same colors as Fig. 2; blue is bidirectional, green is t-h, and red is h-t; $n=7$ fish). Scale bar, $100 \mu \mathrm{m}$. C, The h-t integrators encode a combination of speed and duration. Top, Average response of integrators to the flow of increasing the simulated distance traveled; labels indicate the length of each stimulus. The third and fourth, as well as fifth and sixth are repeats of the stimulus (respectively, the fifth and last $h-t$ stimuli, and the second and eighth from $A$ ). Below are the average areas under the curves for the different conditions ( $n=7$ fish, mean \pm SD). The only significant differences were between the first or second stimulus and the last two or last three stimuli, respectively (adjusted $p$ values for Dunn's test: first vs sixth $=0.0108$; first vs seventh $=0.0444$; second vs fifth $=0.00,444$; second vs sixth $=0.0004$; second vs seventh $=0.0022$ ). Bottom, Visualization of their localization. Rotation in Multimedia 4. $D$, Speed encoding of the flow speed. Top, Average response of the two categories of speed-encoding neurons, either $1 \mathrm{~mm} / \mathrm{s} \mathrm{specific} \mathrm{(blue)} \mathrm{or} 2 \mathrm{~mm} / \mathrm{s}$ specific (red). Bottom, Visualization of their localization. Rotation in Multimedia 5. 3-dimensional rotations of the dorsal views shown in $\mathbf{C}$ and $\boldsymbol{D}$ can be found in Movies 4 and 5 , respectively.

across seven larvae), and the other with twofold stronger response to 1 than $2 \mathrm{~mm} / \mathrm{s}$ (598 ROIs across seven larvae). Although these two clusters also responded to t-h flow, their responses to t-h flow were not speed sensitive. The ROIs belonging to these clusters were mostly found in the MON, the hindbrain, and the TS, with some scattered in other brain regions (Fig. $7 D$ ). The $1 \mathrm{~mm} / \mathrm{s}$ responses were clustered in the middle of the MON, while the 2 $\mathrm{mm} / \mathrm{s}$ responses were at the rostral and caudal edges of the MON. 
Table 3. Tail beat frequency during flow stimulation

\begin{tabular}{llllll}
\hline & Baseline & h-t 1 mm/s & h-t 2 mm/s & t-h $1 \mathrm{~mm} / \mathrm{s}$ & t-h 2 mm/s \\
\hline Fish 1 & 0.154 & 0.224 & 0.447 & 0.229 & 0.360 \\
Fish 2 & 0.356 & 0.336 & 0.054 & 0.135 & 0.147 \\
Fish 3 & 0.420 & 0.658 & 0.677 & 0.390 & 0.893 \\
Fish 4 & 0.709 & 0.546 & 0.393 & 0.417 & 0.200 \\
Fish 5 & 0.699 & 0.378 & 0.596 & 0.283 & 0.426 \\
Mean & 0.467 & 0.428 & 0.433 & 0.291 & 0.405 \\
SD & 0.212 & 0.154 & 0.215 & 0.104 & 0.264 \\
\hline
\end{tabular}

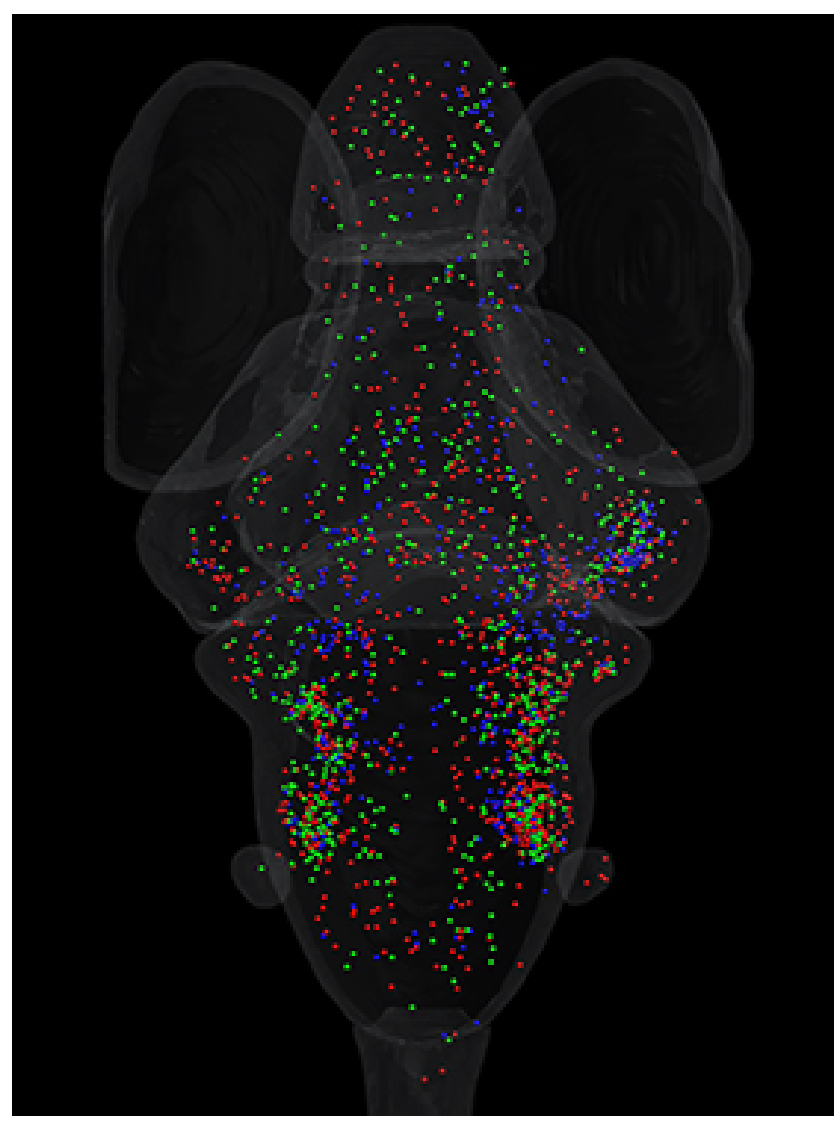

Movie 1. A 3-dimensional rotation of the dorsal view shown in Figure 2A. [View online]

Finally, to ensure that the observed responses were sensory and not motor responses, we imaged fish exposed to the complex stimulus. We embedded the fish in the same manner that they were embedded for the calcium-imaging experiment, but without tubocurarine treatment. We did not observe any significant changes in tail movements during stimulation compared with baseline (Table 3 ), and those movements were also not significantly correlated to the stimuli (Tables 4, 5). We speculate that the flow speed to which our larvae were exposed may be too slow to elicit significant movements. These results show that most of our observed water flow responses were sensory, rather than premotor or motor commands. An alternative explanation lies in the recent observation that the glia may silence futile behaviors in embedded larval zebrafish; the fish may eventually give up swimming during our settling period before imaging can begin (Mu et al., 2019).

\section{Discussion}

Overview of flow-responsive neurons across the brain By performing brain-wide calcium imaging during controlled water flow stimulation, we have identified various categories of
Table 4. Correlation of motor activity to flow stimulation

\begin{tabular}{lrlrrr}
\hline & \multicolumn{1}{c}{ Baseline } & h-t 1 mm/s & h-t 2 mm/s & t-h $1 \mathrm{~mm} / \mathrm{s}$ & t-h 2 mm/s \\
\hline Fish 1 & $-2.65 \mathrm{E}-04$ & $1.43 \mathrm{E}-04$ & $-1.50 \mathrm{E}-04$ & $-8.96 \mathrm{E}-05$ & $1.64 \mathrm{E}-04$ \\
Fish 2 & $2.17 \mathrm{E}-04$ & $4.20 \mathrm{E}-04$ & $-6.92 \mathrm{E}-04$ & $-3.32 \mathrm{E}-04$ & $-2.04 \mathrm{E}-04$ \\
Fish 3 & $-3.06 \mathrm{E}-04$ & $5.30 \mathrm{E}-05$ & $2.20 \mathrm{E}-05$ & $1.18 \mathrm{E}-04$ & $-5.87 \mathrm{E}-04$ \\
Fish 4 & $-2.65 \mathrm{E}-04$ & $1.43 \mathrm{E}-04$ & $-1.50 \mathrm{E}-04$ & $-8.96 \mathrm{E}-05$ & $1.64 \mathrm{E}-04$ \\
Fish 5 & $2.17 \mathrm{E}-04$ & $4.20 \mathrm{E}-04$ & $-6.92 \mathrm{E}-04$ & $-3.32 \mathrm{E}-04$ & $-2.04 \mathrm{E}-04$ \\
Mean & $-9.21 \mathrm{E}-05$ & $2.31 \mathrm{E}-04$ & $-1.26 \mathrm{E}-04$ & $-7.68 \mathrm{E}-05$ & $-5.16 \mathrm{E}-04$ \\
SD & $2.35 \mathrm{E}-04$ & $2.13 \mathrm{E}-04$ & $3.35 \mathrm{E}-04$ & $1.74 \mathrm{E}-04$ & $8.48 \mathrm{E}-04$ \\
\hline
\end{tabular}

Table 5. Pearson's correlations of neural decoding of complex features by the forward integrators

\begin{tabular}{lccc}
\hline \multicolumn{1}{c}{ Model } & Speed & Time & Distance \\
\hline Wiener filter (linear) & $0.214 \pm 0.142$ & $0.394 \pm 0.045$ & $0.459 \pm 0.142$ \\
Wiener cascade (nonlinear) & $0.147 \pm 0.121$ & $0.397 \pm 0.060$ & $0.470 \pm 0.079$ \\
Dense neural network & $0.100 \pm 0.084$ & $0.480 \pm 0.060$ & $0.534 \pm 0.070$ \\
\hline
\end{tabular}

Data are mean $\pm S D$

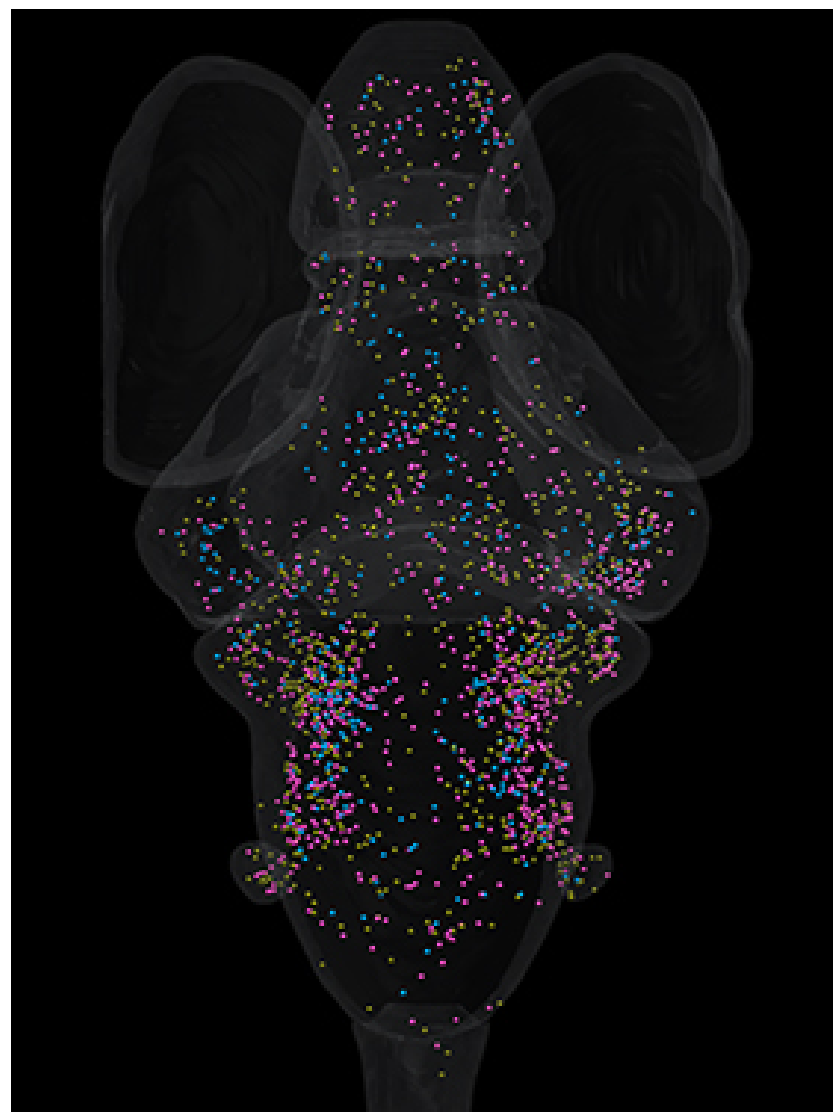

Movie 2. A 3-dimensional rotation of the dorsal view shown in Figure 2B. [View online]

responsive neurons, the stimulus features that they are attuned to, and their distributions across the brain. This allows several observations about the structure and functional architecture of the water flow-sensing network of the brain. We have found that the direction of flow is already represented in the pLLG, but that none of the more complex stimulus properties (onset or total accumulated flow) are represented in this structure. This observation, which is consistent with past electrophysiological recordings of LLG neurons (Liao and Haehnel, 2012; Haehnel-Taguchi et al., 2014), suggests that these simple directional signals provide the initial input for all subsequent water flow processing in the brain. 


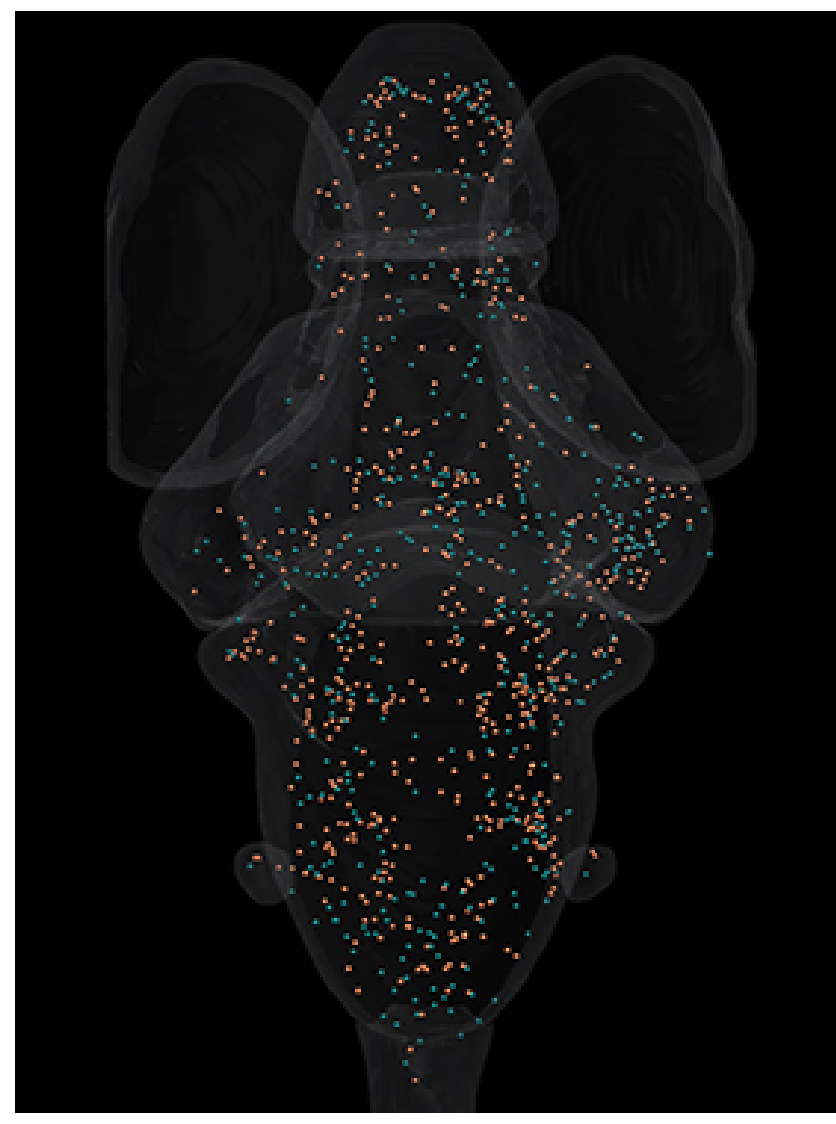

Movie 3. A 3-dimensional rotation of the dorsal view shown in Figure 2C. [View online]

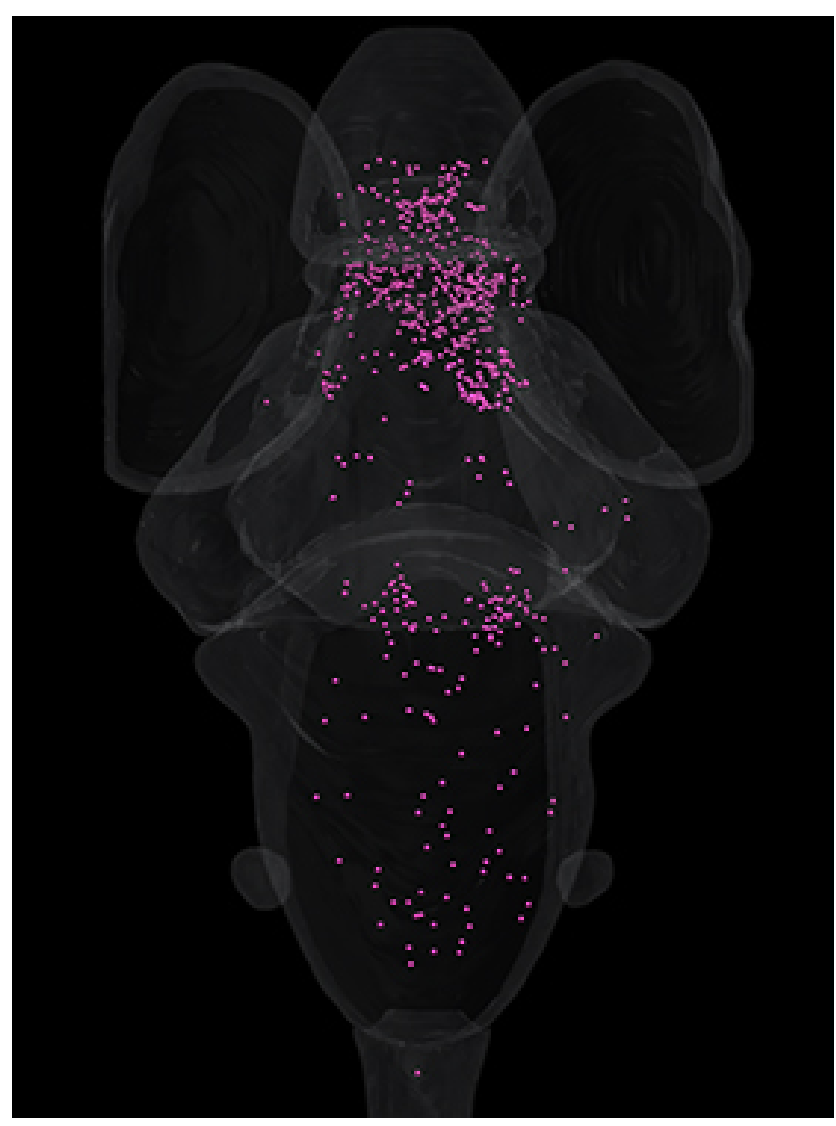

Movie 4. A 3-dimensional rotation of the dorsal view shown in Figure 7C. [View online]

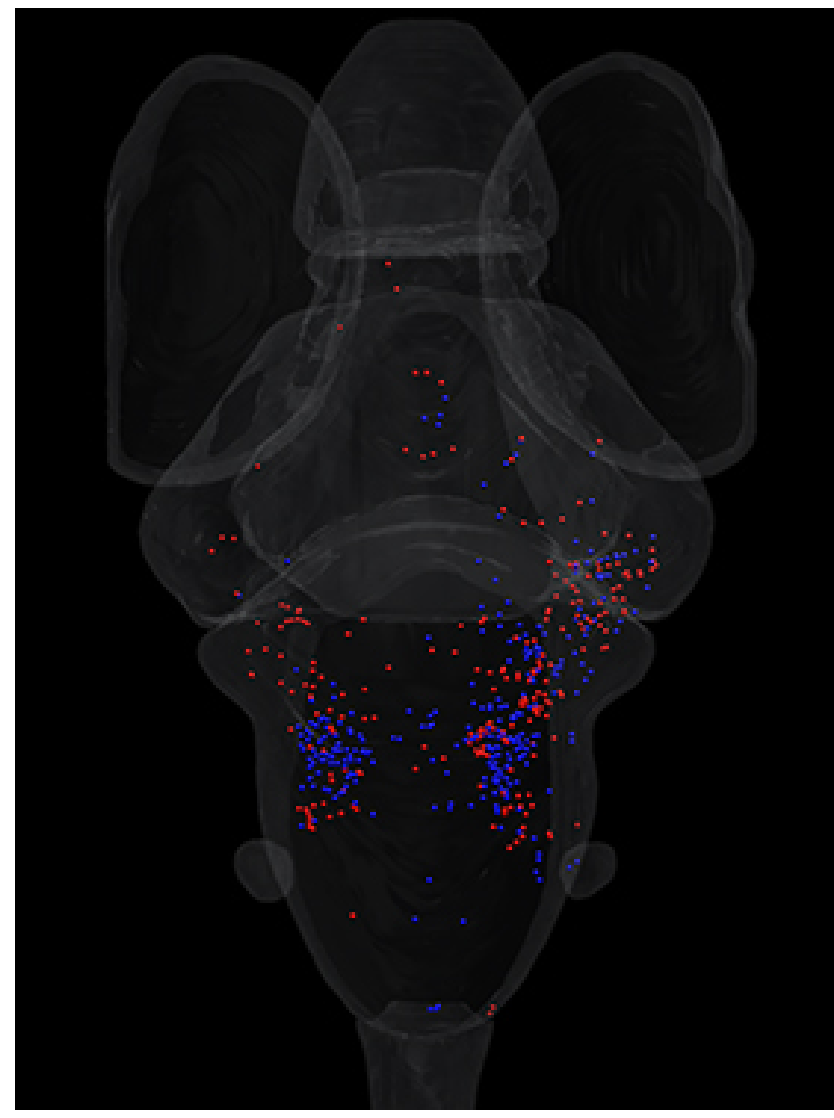

Movie 5. A 3-dimensional rotation of the dorsal view shown in Figure 7D. [View online]

The afferent neurons from the pLLG all project to the MON, where we found second-order neurons attuned to all of the flow stimulus properties (presence, direction, onset, and total volume) identified in our study. These diverse response types observed in the MON are intermingled, showing no spatial organization, consistent with the observations that these projections may be topographically, but not functionally, segregated (Faucherre et al., 2009; Liao, 2010; Liao and Haehnel, 2012; Pujol-Martí et al., 2012). Topographical organization of the flow-sensitive structures, with some units showing tight receptive fields and with individual neuromasts represented in restricted areas of the MON (Plachta et al., 1999), would not have been revealed with our setup, which stimulated all trunk neuromasts simultaneously.

We also observed nuanced responses to water flow stimuli in structures downstream of the MON, including the OT, TS, cerebellum, and telencephalon (Fig. 4). These downstream regions are well positioned to integrate water flow stimuli with other modalities such as visual, vestibular, or auditory (Thompson et al., 2016; Vanwalleghem et al., 2017; Favre-Bulle et al., 2018; Constantin et al., 2019). These telencephalic responses may also be involved in decision-making, perhaps through the algorithm described in the study by Oteiza et al. (2017) to align with the flow (rheotaxis). Another region rich in flow-responsive neurons, the anterior hindbrain, has been recently identified as a central location for evidence accumulation and decision-making (Bahl and Engert, 2020). Overall, this shows how the fish could use the downstream regions to integrate the water flow stimuli with other sensory stimuli to inform its behavioral choices. Future studies could combine stimuli from multiple modalities with different saliencies to assess the precise function that each region would play in their processing and integration. 


\section{Possible neural mechanisms for speed detection and integration}

Our initial observation of integrator neurons was consistent with their representing the accumulation of flow across either time or distance, and our initial stimulus train did not allow us to distinguish between these possibilities. Using a more complex stimulus train, we found evidence for an increased GCaMP response for given neurons at particular speeds (Fig. $7 C$ ), which may indicate that the firing rate in these neurons encodes flow speed (Chen et al., 2013). As the fast-encoding ROIs respond (weakly) to the $1 \mathrm{~mm} / \mathrm{s}$ stimuli, it seems unlikely that these are simply high-threshold ROIs, and this suggests that these signals may actually encode speed. Flow speed could also be represented through temporal coding or coincidence detectors (Mogdans and Bleckmann, 2012), but the temporal kinetics of GCaMP would not have permitted this observation in our preparation. In the future, fluorescent voltage sensors or traditional electrophysiology may allow such observations to be made (Akemann et al., 2010).

The integrators that we have described match nicely with the type of information that a recently developed rheotaxis algorithm would require, in which the accumulated flow across the two sides of the body is compared with drive-turning behavior (Oteiza et al., 2017). We showed that these integrators do not simply encode the duration of the stimulus, but a combination of time and flow speed. The size of the zebrafish larva and the design of our microfluidics chamber precluded the presentation of lateralized stimuli, but such an experiment in the future could test this rheotaxis model concretely (Oteiza et al., 2017).

\section{Coding of detailed information about $h-t$ water flow}

By several measures, the brain-wide water flow network appears to be slanted toward the detection and complex processing of water flow like that experienced by a forward-swimming larva. This evidence includes a greater number of h-t-responsive neurons (Fig. 1), more pronounced integration of h-t flow stimuli (Figs. 2, 4), speedencoding neurons for h-t flow (Fig. 7), and greater functional involvement of h-t-responsive neurons (as measured by degree and participation) in correlation-based network graphs (Fig. 6). Indeed, even the bidirectional response profiles that we have described were slanted toward h-t responsiveness (Fig. 2D).

Combined, these observations suggest that larvae are sensitive to the presence or the onset of water flow in the t-h direction, which may be sufficient, for instance, to detect the water flow produced by the attack of a suction predator. A more nuanced appreciation of the details of h-t flow may subserve not only evasive behaviors, but also the fine control of swimming and navigation. The h-t flow is also the perceived flow direction when fish swim to align with the flow during rheotaxis to remain in place (Olszewski et al., 2012; Oteiza et al., 2017). Viewed this way, the emphasis on h-t flow processing that we observe is unsurprising, since this type of flow would be more common, and likely more ethologically relevant, to larval zebrafish. Recent observations of specific silencing of the h-t flow response at the neuromast level by efferent copies during self-motion also support an ethological relevance to the h-t flow (Pichler and Lagnado, 2020). However, the paralyzing agent we used blocks the cholinergic receptors that mediate this silencing by the motor neurons, and we did not observe swim bouts as a result of our stimuli. How this silencing interacts with our observed responses and how the brain still manages to make the relevant computations would be an interesting topic for future study.
It is also possible that we are underestimating this bias toward perception of a simulated forward movement, given our imaging setup. Since we are using a head-embedded preparation, we are stimulating only the trunk lateral line. Because we are not stimulating the cranial neuromasts, we may be missing responses that would presumably, based on their position on the body of the animal, report on h-t flow. We may also be missing more specialized functions of the cranial neuromasts, such as the use of flow or vibrational information during predatory behavior (Pohlmann et al., 2004; Carrillo and McHenry, 2016).

The encoding and processing of the water flow stimuli may change with age, as the ratio of body size to stimulus features changes, and with the development of canal neuromasts (Van Trump and McHenry, 2008; Wada et al., 2014). As such, studying the ontogeny of the processing would be interesting, especially for the afferent neurons receiving information from the newly developed canal neuromasts, which have been linked to foraging in zebrafish (Carrillo et al., 2019). However, collecting such an ontological dataset would require imaging in ossified zebrafish, whose skulls and pigmentation preclude the use of one-photon light sheet microscopy. Danionella translucida, a promising new fish model, allows imaging of the adult brain and could thus be used for such a developmental study of the lateral line sensory network (Schulze et al., 2018).

To our knowledge, this is the first brain-wide study of lateral line information processing. By observing activity across the brain at cellular resolution, we have uncovered multiple classes of responses representing several features of the flow, and have located each of these response types to its position in the brain. While these results are, in a sense, a comprehensive accounting of lateral line processing in this system, such studies leave many open questions about the circuit-level mechanisms governing the network (Vanwalleghem et al., 2018). The use of GCaMP prevents our judging the order in which events occur across the network, making it difficult to infer the direction of information flow. We also do not know the structures or connectivity of the neurons whose signals we are detecting, and this prevents an anatomic validation of our correlation-based network that we have described using graph theory. In the future, registration of these response types to the morphologies of associated neurons, for instance using databases of neurons across the larval zebrafish brain (Kunst et al., 2019), would allow our activity-based models to be grounded in a plausible anatomic framework. Registration against electron microscopy data could allow us to infer connectivity within the network at the synaptic level (Hildebrand et al., 2017).

More sophisticated stimuli, targeting individual neuromasts or temporally controlled combinations of neuromasts, will be necessary to reveal topography or the computations that allow larvae to detect complex real-world flow patterns. Finally, future functional work, likely using optogenetics, will be necessary to test the resulting models of information flow concretely. Viewed from this perspective, the current study provides a first tantalizing glimpse of this brain-wide network and a departure point for targeted studies of its structural and mechanistic details.

\section{References}

Akemann W, Mutoh H, Perron A, Rossier J, Knöpfel T (2010) Imaging brain electric signals with genetically targeted voltage-sensitive fluorescent proteins. Nat Methods 7:643-649.

Alexandre D, Ghysen A (1999) Somatotopy of the lateral line projection in larval zebrafish. Proc Natl Acad Sci U S A 96:7558-7562. 
Ali R, Mogdans J, Bleckmann H (2010) Responses of medullary lateral line units of the goldfish, Carassius auratus, to amplitude-modulated sinusoidal wave stimuli. Int J Zool 2010:1-14.

Avants BB, Yushkevich P, Pluta J, Minkoff D, Korczykowski M, Detre J, Gee JC (2010) The optimal template effect in hippocampus studies of diseased populations. Neuroimage 49:2457-2466.

Avants BB, Tustison NJ, Song G, Cook PA, Klein A, Gee JC (2011) A reproducible evaluation of ANTs similarity metric performance in brain image registration. Neuroimage 54:2033-2044.

Bahl A, Engert F (2020) Neural circuits for evidence accumulation and decision making in larval zebrafish. Nat Neurosci 23:94-102.

Bleckmann H (2008) Peripheral and central processing of lateral line information. J Comp Physiol A Neuroethol Sens Neural Behav Physiol 194:145-158.

Butler JM, Maruska KP (2015) The mechanosensory lateral line is used to assess opponents and mediate aggressive behaviors during territorial interactions in an African cichlid fish. J Exp Biol 218:3284-3294.

Carrillo A, McHenry MJ (2016) Zebrafish learn to forage in the dark. J Exp Biol 219:582-589.

Carrillo A, Van Le D, Byron ML, Jiang H, McHenry M (2019) Canal neuromasts enhance foraging in zebrafish (Danio rerio). Bioinspir Biomim 14:035003.

Chen TW, Wardill TJ, Sun Y, Pulver SR, Renninger SL, Baohan A, Schreiter ER, Kerr RA, Orger MB, Jayaraman V, Looger LL, Svoboda K, Kim DS (2013) Ultrasensitive fluorescent proteins for imaging neuronal activity. Nature 499:295-300.

Constantin L, Poulsen RE, Favre-Bulle IA, Taylor MA, Sun B, Goodhill GJ, Vanwalleghem GC, Scott 4 (2019) Altered brain-wide auditory networks in mutant larval zebrafish. bioRxiv. doi:10.1101/722082.

Dijkgraaf S (1963) The functioning and significance of the lateral-line organs. Biol Rev Camb Philos Soc 38:51-105.

Edelstein A, Amodaj N, Hoover K, Vale R, Stuurman N (2010) Computer control of microscopes using microManager. Curr Protoc Mol Biol Chapter14:Unit14.20.

Faucherre A, Pujol-Martí J, Kawakami K, López-Schier H (2009) Afferent neurons of the zebrafish lateral line are strict selectors of hair-cell orientation. PLoS One 4:e4477.

Favre-Bulle IA, Stilgoe AB, Rubinsztein-Dunlop H, Scott EK (2017) Optical trapping of otoliths drives vestibular behaviours in larval zebrafish. Nat Commun 8:630

Favre-Bulle IA, Vanwalleghem G, Taylor MA, Rubinsztein-Dunlop H, Scott EK (2018) Cellular-resolution imaging of vestibular processing across the larval zebrafish brain. Curr Biol 28:3711.e3-3722.e3.

Ghysen A, Dambly-Chaudière C (2004) Development of the zebrafish lateral line. Curr Opin Neurobiol 14:67-73.

Glaser JI, Benjamin AS, Chowdhury RH, Perich MG, Miller LE, Kording KP (2017) Machine learning for neural decoding. arXiv. Advance online publication. Retrieved April 13, 2020. doi:10.1708.00909.

Grama A, Engert F (2012) Direction selectivity in the larval zebrafish tectum is mediated by asymmetric inhibition. Front Neural Circuits 6:59.

Haehnel-Taguchi M, Akanyeti O, Liao JC (2014) Afferent and motoneuron activity in response to single neuromast stimulation in the posterior lateral line of larval zebrafish. J Neurophysiol 112:1329-1339.

Harris JA, Cheng AG, Cunningham LL, MacDonald G, Raible DW, Rubel EW (2003) Neomycin-induced hair cell death and rapid regeneration in the lateral line of zebrafish (Danio rerio). J Assoc Res Otolaryngol 4:219-234.

Hildebrand DGC, Cicconet M, Torres RM, Choi W, Quan TM, Moon J, Wetzel AW, Scott Champion A, Graham BJ, Randlett O, Plummer GS, Portugues R, Bianco IH, Saalfeld S, Baden AD, Lillaney K, Burns R, Vogelstein JT, Schier AF, Lee W-CA, et al. (2017) Whole-brain serial-section electron microscopy in larval zebrafish. Nature 545:345-349.

Ji YR, Warrier S, Jiang T, Wu DK, Kindt KS (2018) Directional selectivity of afferent neurons in zebrafish neuromasts is regulated by Emx2 in presynaptic hair cells. Elife 7:e35796.

Kunst M, Laurell E, Mokayes N, Kramer A, Kubo F, Fernandes AM, Forster D, Dal Maschio M, Baier H (2019) A cellular-resolution atlas of the larval zebrafish brain. Neuron 103:21.e5-38.e5.

Künzel S, Bleckmann H, Mogdans J (2011) Responses of brainstem lateral line units to different stimulus source locations and vibration directions. J Comp Physiol A Neuroethol Sens Neural Behav Physiol 197:773-787.

Liao JC (2010) Organization and physiology of posterior lateral line afferent neurons in larval zebrafish. Biol Lett 6:402-405.
Liao JC, Haehnel M (2012) Physiology of afferent neurons in larval zebrafish provides a functional framework for lateral line somatotopy. J Neurophysiol 107:2615-2623.

Marquart GD, Tabor KM, Horstick EJ, Brown M, Geoca AK, Polys NF, Nogare DD, Burgess HA (2017) High-precision registration between zebrafish brain atlases using symmetric diffeomorphic normalization. Gigascience 6:1-15.

McCormick CA (1989) Central lateral line mechanosensory pathways in bony fish. In: The mechanosensory lateral line: neurobiology and evolution. (Coombs S, Görner P, Münz H, eds), pp 341-364. New York: Springer.

McCormick CA, Hernandez DV (1996) Connections of octaval and lateral line nuclei of the medulla in the goldfish, including the cytoarchitecture of the secondary octaval population in goldfish and catfish. Brain Behav Evol 47:113-137.

McHenry MJ, Feitl KE, Strother JA, Van Trump WJ (2009) Larval zebrafish rapidly sense the water flow of a predator's strike. Biol Lett 5:477-479.

Metcalfe WK, Kimmel CB, Schabtach E (1985) Anatomy of the posterior lateral line system in young larvae of the zebrafish. J Comp Neurol 233:377-389.

Mogdans J, Bleckmann H (2012) Coping with flow: behavior, neurophysiology and modeling of the fish lateral line system. Biol Cybern 106:627-642.

Mogdans J, Bleckmann H, Menger N (1997) Sensitivity of central units in the goldfish, Carassius auratus, to transient hydrodynamic stimuli. Brain Behav Evol 50:261-283.

Montgomery JC, Baker CF, Carton AG (1997) The lateral line can mediate rheotaxis in fish. Nature 389:960-963.

Mu Y, Bennett DV, Rubinov M, Narayan S, Yang CT, Tanimoto M, Mensh BD, Looger LL, Ahrens MB (2019) Glia accumulate evidence that actions are futile and suppress unsuccessful behavior. Cell 178:27.e19-43.e19.

Nagiel A, Andor-Ardó D, Hudspeth AJ (2008) Specificity of afferent synapses onto plane-polarized hair cells in the posterior lateral line of the zebrafish. J Neurosci 28:8442-8453.

Olszewski J, Haehnel M, Taguchi M, Liao JC (2012) Zebrafish larvae exhibit rheotaxis and can escape a continuous suction source using their lateral line. PLoS One 7:e36661.

Oteiza P, Odstrcil I, Lauder G, Portugues R, Engert F (2017) A novel mechanism for mechanosensory-based rheotaxis in larval zebrafish. Nature 547:445-448.

Partridge BL, Pitcher TJ (1980) The sensory basis of fish schools: relative roles of lateral line and vision. J Comp Physiol 135:315-325.

Pichler P, Lagnado L (2019) The transfer characteristics of hair cells encoding mechanical stimuli in the lateral line of zebrafish. J Neurosci 39:112-124.

Pichler P, Lagnado L (2020) Motor behavior selectively inhibits hair cells activated by forward motion in the lateral line of zebrafish. Curr Biol 30:150.e3-157.e3.

Plachta D, Mogdans J, Bleckmann H (1999) Responses of midbrain lateral line units of the goldfish, Carassius auratus, to constant-amplitude and amplitude-modulated water wave stimuli. J Comp Physiol A Neuroethol Sens Neural Behav Physiol 185:405-417.

Pnevmatikakis EA, Soudry D, Gao Y, Machado TA, Merel J, Pfau D, Reardon T, Mu Y, Lacefield C, Yang W, Ahrens M, Bruno R, Jessell TM, Peterka DS, Yuste R, Paninski L (2016) Simultaneous denoising, deconvolution, and demixing of calcium imaging data. Neuron 89:285-299.

Pohlmann K, Atema J, Breithaupt T (2004) The importance of the lateral line in nocturnal predation of piscivorous catfish. J Exp Biol 207:2971-2978.

Pujol-Martí J, Zecca A, Baudoin J-P, Faucherre A, Asakawa K, Kawakami K, López-Schier H (2012) Neuronal birth order identifies a dimorphic sensorineural map. J Neurosci 32:2976-2987.

Raible DW, Kruse GJ (2000) Organization of the lateral line system in embryonic zebrafish. J Comp Neurol 421:189-198.

Randlett O, Wee CL, Naumann EA, Nnaemeka O, Schoppik D, Fitzgerald JE, Portugues R, Lacoste AM, Riegler C, Engert F, Schier AF (2015) Wholebrain activity mapping onto a zebrafish brain atlas. Nat Methods 12:1039-1046.

Rubinov M, Sporns O (2010) Complex network measures of brain connectivity: uses and interpretations. Neuroimage 52:1059-1069.

Schulze L, Henninger J, Kadobianskyi M, Chaigne T, Faustino AI, Hakiy N, Albadri S, Schuelke M, Maler L, Del Bene F, Judkewitz B (2018) Transparent Danionella translucida as a genetically tractable vertebrate brain model. Nat Methods 15:977-983.

Schuster K, Dambly-Chaudière C, Ghysen A (2010) Glial cell line-derived neurotrophic factor defines the path of developing and regenerating axons in the lateral line system of zebrafish. Proc Natl Acad Sci U S A 107:19531-19536 
Severi KE, Portugues R, Marques JC, O’Malley DM, Orger MB, Engert F (2014) Neural control and modulation of swimming speed in the larval zebrafish. Neuron 83:692-707.

Suli A, Watson GM, Rubel EW, Raible DW (2012) Rheotaxis in larval zebrafish is mediated by lateral line mechanosensory hair cells. PLoS One 7: e29727.

Taylor MA, Vanwalleghem GC, Favre-Bulle IA, Scott EK (2018) Diffuse light-sheet microscopy for stripe-free calcium imaging of neural populations. J Biophotonics 11:e201800088.

Theiler J, Eubank S, Longtin A, Galdrikian B, Doyne Farmer J (1992) Testing for nonlinearity in time series: the method of surrogate data. Physica D 58:77-94.

Thévenaz P, Ruttimann UE, Unser M (1998) A pyramid approach to subpixel registration based on intensity. IEEE Trans Image Process 7:27-41.

Thielicke W, Stamhuis E (2014) PIVlab-towards user-friendly, affordable and accurate digital particle image velocimetry in MATLAB. J Open Res Softw 2:e30.
Thompson AW, Vanwalleghem GC, Heap LA, Scott EK (2016) Functional profiles of visual-, auditory-, and water flow-responsive neurons in the zebrafish tectum. Curr Biol 26:743-754

Van Trump WJ, McHenry MJ (2008) The morphology and mechanical sensitivity of lateral line receptors in zebrafish larvae (Danio rerio). J Exp Biol 211:2105-2115.

Vanwalleghem G, Heap LA, Scott EK (2017) A profile of auditory-responsive neurons in the larval zebrafish brain. J Comp Neurol 525:3031-3043.

Vanwalleghem GC, Ahrens MB, Scott EK (2018) Integrative whole-brain neuroscience in larval zebrafish. Curr Opin Neurobiol 50:136-145.

Wada H, Iwasaki M, Kawakami K (2014) Development of the lateral line canal system through a bone remodeling process in zebrafish. Dev Biol 392:1-14.

Westerfield M (2000) The zebrafish book. A guide for the laboratory use of zebrafish (Danio rerio), Ed 4. Eugene, OR: University of Oregon.

Wullimann MF (1997) The physiology of fishes, Ed 2. Abingdon, UK: Taylor and Francis. 